\title{
INTERSECTION EXPONENTS FOR PLANAR BROWNIAN MOTION
}

\author{
By Gregory F. LAWLeR ${ }^{1}$ AND Wendelin WeRner \\ Duke University and Université Paris-Sud
}

\begin{abstract}
We derive properties concerning all intersection exponents for planar Brownian motion and we define generalized exponents that, loosely speaking, correspond to noninteger numbers of Brownian paths. Some of these properties lead to general conjectures concerning the exact value of these exponents.
\end{abstract}

1. Introduction. The main goal of this paper is to derive some properties of intersection exponents for two-dimensional Brownian motions. These new properties improve our understanding of these exponents and lead to general conjectures concerning their exact values.

Suppose that $n+p$ independent planar Brownian motion $\beta^{1}, \ldots, \beta^{n}$ and $\gamma^{1}, \ldots, \gamma^{p}$ are started from points $\beta_{0}^{1}=\cdots=\beta_{0}^{n}=(1,1)$ and $\gamma_{0}^{1}=\cdots=\gamma_{0}^{p}$ $=(2,1)$ in the complex plane, and consider the probability that for all $j \leq n$ and $l \leq p$, the paths of $\beta^{j}$ up to time $t$ and of $\gamma^{l}$ up to time $t$ do not intersect; more precisely,

$$
f_{n, p}(t)=\mathbf{P}\left(\bigcup_{j=1}^{n} \beta^{j}[0, t] \cap \bigcup_{l=1}^{p} \gamma^{l}[0, t]=\varnothing\right) .
$$

It is easy to see that this probability decays as $t \rightarrow \infty$ roughly (i.e., logarithmically speaking) like a power of $t$. The $(n, p)$-intersection exponent $\xi(n, p)$ is defined as being twice this power, that is,

$$
f_{n, p}(t) \approx t^{-\xi(n, p) / 2}, \quad t \rightarrow \infty
$$

[we write $a(t) \approx b(t)$ for $\log (a(t)) \sim \log (b(t))$ ]. We say that $\xi(n, p)$ is the intersection exponent between one packet of $n$ Brownian motions and one packet of $p$ Brownian motions.

Similarly, one can define more general intersection exponents between $k \geq 2$ packets of Brownian motions containing, respectively, $p_{1}, p_{2}, \ldots, p_{k}$ paths. Each path of the $j$ th packet starts from $(j, 1)$ and has to avoid all paths of all other packets. We denote by $\xi\left(p_{1}, p_{2}, \ldots, p_{k}\right)$ the corresponding exponent.

As shown by the first author in [17, 19, 21], several of these exponents correspond to Hausdorff dimensions of exceptional subsets of the planar

Received November 1998; revised April 1999.

${ }^{1}$ Supported in part by the National Science Foundation.

AMS 1991 subject classifications. Primary 60J65; secondary 81T40.

Key words and phrases. Brownian motion, critical exponents, conformal invariance. 
Brownian curve. For instance (see [17]), the Hausdorff dimension of the set of cut points of a planar Brownian path is $2-\xi(1,1)$.

A crucial role in this paper will be played by intersection exponents in a half-space. Those are defined exactly as the intersection exponents above except that one adds the condition that all Brownian motion paths (up to time $t$ ) remain in the upper half-plane $H=\{(x, y): y>0\}$ (this is the reason why we chose the starting points in $H)$. We call these exponents $\tilde{\xi}\left(p_{1}, \ldots, p_{k}\right)$. For instance, $\tilde{\xi}(1,1)$ is defined by

$\mathbf{P}\left(\beta^{1}[0, t] \cap \gamma^{1}[0, t]=\varnothing, \beta^{1}[0, t] \cup \gamma^{1}[0, t] \subset H\right) \approx t^{-\tilde{\xi}(1,1) / 2}, \quad t \rightarrow \infty$.

Physicists (see [10]) have made the striking conjecture that many of these exponents are rational numbers (just as in many other two-dimensional statistical physics models). Except for the special value $\xi(2,1)=2$ (see [15]), exact values of $\xi$ 's and $\tilde{\xi}$ 's are not known rigorously. For conjectures, see [10], [11]; for some rigorous bounds, see [5], [33], [34]; for some simulations, see [6], [10], [26], [30]. Note that analogous exponents can be defined for simple planar random walks; it can be shown that the exponents for Brownian motion and random walks are the same; see [4], [8], [24], [25].

The main results of the present paper can be summed up as follows (even though they are not being derived in that order).

1. We give a precise natural meaning to the exponents $\xi\left(\mu_{1}, \ldots, \mu_{k}\right)$ and $\tilde{\xi}\left(\lambda_{1}, \ldots, \lambda_{k}\right)$ for all positive real numbers $\lambda_{1}, \ldots, \lambda_{k}, \mu_{1}, \ldots, \mu_{k}$ with

$$
\mu_{i} \geq 1 \text { and } \mu_{i^{\prime}} \geq 1 \text { for some } i \neq i^{\prime}
$$

(i.e., two of the $\mu_{i}$ 's are not smaller than one). These definitions generalize the above definitions in the case where the $\lambda$ 's are integers. Hence, the exponents can be seen as values of real functions at integer points.

2 . We show that these functions satisfy certain functional relations. First, for all $2 \leq j \leq k$ and for all positive $\lambda_{1}, \ldots, \lambda_{k}$ one has the following relation:

$$
\tilde{\xi}\left(\lambda_{1}, \ldots, \lambda_{j-1}, \tilde{\xi}\left(\lambda_{j}, \ldots, \lambda_{k}\right)\right)=\tilde{\xi}\left(\lambda_{1}, \ldots, \lambda_{k}\right)
$$

For instance,

$$
\tilde{\xi}(1,1,1)=\tilde{\xi}(1, \tilde{\xi}(1,1)) .
$$

Similarly, for all $\mu_{1}, \ldots, \mu_{k}$ such that $\mu_{i} \geq 1$ and $\mu_{i^{\prime}} \geq 1$ for some $i \neq i^{\prime}$ and $i<j$ :

$$
\xi\left(\mu_{1}, \ldots, \mu_{j-1}, \tilde{\xi}\left(\mu_{j}, \ldots, \mu_{k}\right)\right)=\xi\left(\mu_{1}, \ldots, \mu_{k}\right)
$$

We call these relations "cascade relations." Second, $\xi$ and $\tilde{\xi}$ satisfy "commutation relations," that is, they are symmetric functions of their arguments (this is not straightforward because our definition of the generalized exponents is not symmetric).

3. One can define a positive, strictly increasing continuous function $U$ on $[0, \infty)$ by

$$
U^{2}(\lambda)=\lim _{N \rightarrow \infty} \frac{\tilde{\xi}\left(\lambda^{\otimes N}\right)}{N^{2}},
$$


where $\lambda^{\otimes N}=(\lambda, \lambda, \lambda, \ldots, \lambda) \in[0, \infty)^{N}$. Then, we derive the following functional relation: for all positive $\lambda_{1}, \ldots, \lambda_{k}$,

$$
U\left(\tilde{\xi}\left(\lambda_{1}, \ldots, \lambda_{k}\right)\right)=U\left(\lambda_{1}\right)+\cdots+U\left(\lambda_{k}\right) .
$$

This shows in particular that the exact values of all half-plane exponents $\tilde{\xi}$ are encoded in the function $U$.

4. Using the previous results, one can then also show that the full-plane exponents $\xi$ can be represented as a function which depends on $\mu_{j}$ 's only through $\tilde{\xi}$; more precisely, there exists a continuous increasing function $\eta$ such that for all $\mu_{1}, \ldots, \mu_{k}$ (such that $\mu_{i} \geq 1$ and $\mu_{i^{\prime}} \geq 1$ for some $\left.i \neq i^{\prime}\right)$

$$
\xi\left(\mu_{1}, \ldots, \mu_{k}\right)=\eta\left(\tilde{\xi}\left(\mu_{1}, \ldots, \mu_{k}\right)\right) .
$$

This function $\eta(\cdot)$ can be interpreted as a generalized disconnection exponent (see, for instance, [33] for a definition of disconnection exponents).

5. Combining these rigorous results with Duplantier-Kwon's [10] conjectures for $\tilde{\xi}\left(1^{\otimes N}\right)$ and $\xi\left(1^{\otimes N}\right)$ leads to a general conjecture for the functions $U$ and $\eta$ (and therefore for all exponents $\xi$ and $\tilde{\xi}$ ) that we state in Section 7 . These conjectures seem to be confirmed by several cross-checks. It is interesting to remark that certain values of the exponents (for integer $\lambda$ 's) turn out to be irrational numbers [for instance $\xi(1,3)$ ]. The conjecture for the exponents $\xi(2, \lambda)$ corresponds to a conjecture for the multifractal spectrum of the Brownian frontier (using the correspondence derived in [21]).

6. Some of the ideas used for Brownian motions lead to conjectures for nonintersection exponents for loop-erased random walks that we state in Section 8.

Duplantier-Kwon's conjectures were derived using nonrigorous (from the mathematician's point of view) considerations using ideas from conformal field theory. One key idea is to admit (for some reasons) that the exponents $\xi\left(1^{\otimes N}\right)$ for integer $N$ 's belong to Kác's table corresponding to some highestweight representations of the Virasoro algebra (see, e.g., [12] for references about Virasoro algebra). This is a purely discrete argument: a discrete set of numbers has to contain another discrete sequence of numbers. Our results and conjectures show that one could look for a continuous function rather than for a discrete sequence.

The results are derived as follows. In Section 2 , we first give a precise tractable definition of the families of exponents $\xi\left(p_{1}, \ldots, p_{k}\right)$ and $\tilde{\xi}\left(p_{1}, \ldots, p_{k}\right)$ (where $p_{1}, \ldots, p_{k}$ are integers). We then construct exponents

$$
\tilde{\xi}\left(\lambda_{1}, p_{1}, \lambda_{2}, p_{2}, \ldots, p_{k}, \lambda_{k+1}\right) \quad \text { and } \xi\left(p_{1}, \lambda_{1}, p_{2}, \lambda_{2}, \ldots, p_{k}, \lambda_{k}\right),
$$

where $p$ 's are positive integers and $\lambda$ 's are nonnegative reals. In the construction we use conditioned Brownian motions rather than Brownian motions; this leads us in the next section to define a measure on paths restricted to a finite domain.

In Section 3, we first recall some general facts concerning conformal invariance and then use these to construct a conformally invariant measure 
on paths in certain domains. We then use this measure to derive a first version of the cascade relations (1) and (2) for the exponents defined so far. In Section 4, we derive the commutation relation that enables us to define all exponents $\xi\left(\mu_{1}, \ldots, \mu_{k}\right)$ and $\tilde{\xi}\left(\lambda_{1}, \ldots, \lambda_{k}\right)$ in such a way that the commutation and cascade relations still hold for these generalized exponents. In Section 5, we study the asymptotic behavior of $\tilde{\xi}\left(\lambda^{\otimes N}\right)$ and show (3) and (4). In Section 6, we use an alternative approach using a rate function to derive asymptotics of the functions $\xi$ and $\tilde{\xi}$. Sections 7 and 8 are devoted to conjectures concerning the precise values of intersection exponents for Brownian motion (in Section 7) and loop-erased random walks (in Section 8).

\section{Intersection exponents.}

2.1. Preliminaries. In order to simplify the reading of the following construction, we first review a few standard facts concerning Brownian motion hitting times and three-dimensional Bessel processes. The reader acquainted with those may as well skip this subsection.

Suppose that $X$ and $Y$ denote two independent (one-dimensional) Brownian motions started from 0 , and define for any stochastic process $\left(Z_{t}, t \geq 0\right)$,

$$
T_{n}(Z)=\inf \left\{t>0: Z_{t}=n\right\}
$$

(with inf $\varnothing=\infty$ ). Then, we have the following well-known estimates [(6) is a consequence of the decomposition of the Dirichlet Laplacian in an interval; see, e.g., [29], page 52; (7) can be found in [31], Proposition II.3.7]: for all $a>0$,

and

$$
\frac{2}{\pi} \exp \left\{-\frac{x}{2 a^{2}}\right\} \leq \mathbf{P}\left(T_{a \pi / 2}(|Y|)>x\right) \leq \frac{4}{\pi} \exp \left\{-\frac{x}{2 a^{2}}\right\}
$$

Hence, if $B=X+i Y$ denotes the complex Brownian motion started from 0 and if $I_{a}=\{z \in \mathbb{C}: \mathfrak{\Im}(z) \in(-a \pi / 2, a \pi / 2)\}$ denotes the horizontal strip of width $a$,

(8) $\frac{2}{\pi} e^{-n / a} \leq \mathbf{P}\left(B\left[0, T_{n}(X)\right] \subset I_{a}\right)=\mathbf{P}\left(T_{a \pi / 2}(|Y|)>T_{n}(X)\right) \leq \frac{4}{\pi} e^{-n / a}$.

It will sometimes be more convenient to work with Brownian motion conditioned to remain in the half-space $\{\Re(z)>0\}$. Let $\hat{X}$ be a three-dimensional Bessel process. It is well known (see, e.g., [31]) that three-dimensional Bessel processes can be viewed as (one-dimensional) Brownian motions "conditioned to stay forever positive." More precisely, let

$$
\hat{T}_{n}=T_{n}(\hat{X})=\inf \left\{t>0: \hat{X}_{t}=n\right\} .
$$

Assume for the moment that $\hat{X}_{0}=a \in(0, n)$. Then, the rigorous version of the previous statement is that the distribution of

$$
\hat{X}_{t}, \quad 0 \leq t \leq \hat{T}_{n},
$$


is the same as the conditional distribution of a Brownian motion starting at $a$,

given the event

$$
X_{t}, \quad 0 \leq t \leq T_{n}(X)
$$

$$
\left\{0 \notin X\left[0, T_{n}(X)\right]\right\} .
$$

Note that this last event has probability $a / n$. From now on, we assume that $\hat{X}_{0}=0$.

There is another well-known feature concerning three-dimensional Bessel processes (see, e.g., again [31]) that will be useful: suppose that $n>m>0$ and define

$$
\hat{\tau}_{m, n}=\sup \left\{t \in\left(0, \hat{T}_{n}\right): \hat{X}_{t}=m\right\} \text { and } \tau_{m, n}=\sup \left\{t \in\left(0, T_{n}\right): X_{t}=m\right\} .
$$

In plain words, $\tau_{m, n}$ (resp., $\hat{\tau}_{m, n}$ ) is the last time $X$ (resp., $(\hat{X})$ crosses the level $m$ before it hits $n$ for the first time. Then, the laws of

$$
\begin{gathered}
\left(\hat{X}_{t}-m, t \in\left[\hat{\tau}_{m, n}, \hat{T}_{n}\right]\right),\left(X_{t}-m, t \in\left[\tau_{m, n}, T_{n}\right]\right) \text { and } \\
\left(\hat{X}_{t}, t \in\left[0, \hat{T}_{n-m}\right]\right)
\end{gathered}
$$

are identical.

Moreover, the three "parts" of the path

$$
\left(\hat{X}_{t}, t \in\left[0, \hat{T}_{m}\right]\right),\left(\hat{X}_{t}, t \in\left[\hat{T}_{m}, \hat{\tau}_{m, n}\right]\right) \text { and }\left(\hat{X}_{t}, t \in\left[\hat{\tau}_{m, n}, \hat{T}_{n}\right]\right)
$$

are independent (the analogous statement for $X$ holds as well).

We will make an extensive use of two-dimensional "conditioned Brownian motion" defined by $W=\hat{X}+i Y$ where $Y$ is one-dimensional Brownian motion independent of $\hat{X}$.

Finally, let us recall that planar Brownian motion is invariant under conformal transformations in the following sense. Suppose that $\left(\gamma_{t}, t \geq 0\right)$ is a planar Brownian motion started from $x \in D$ ( $D$ is an open subset of $\mathbb{C}$ ) and that $D$ is mapped onto $D^{\prime}$ by a conformal map $\Phi$. Then the law of $\left(\Phi\left(\gamma_{t}\right)\right.$, $t \geq 0)$ is that of a time-changed Brownian motion started from $\Phi(x)$ and killed when it exits $D^{\prime}$. In particular, the law of the trace $\left\{\Phi\left(\gamma_{t}\right), t \geq 0\right\}$ is exactly that of the trace of a planar Brownian motion started from $\Phi(x)$ and killed when it exits $\Phi(D)$.

Suppose for instance that $\left(B_{t}, t \geq 0\right)$ is a planar Brownian motion started on the imaginary line from the point $z \in i \mathbb{R}$ and stopped at the time

$$
T_{n}=\inf \left\{t>0: \mathfrak{R}\left(B_{t}\right)=n\right\} .
$$

Then the law of

$$
\left\{\exp \left(B_{t}\right), 0 \leq t \leq T_{n}\right\}
$$

is that of the trace of a planar Brownian motion started from $e^{z}$ (on the unit circle) and killed at the first time it hits the circle of radius $\exp (n)$. We will often implicitly use such facts. 
2.2. Exponents for unconditioned Brownian motions. In this section we define and review some facts about the intersection exponent(s) in two dimensions. Actually, at this stage, it will not be clear whether the exponents that we will define in this section are indeed the same as those briefly defined in the introduction. This will be in fact an easy consequence of the commutation relations stated in Section 4.

Let $B_{t}^{1}, B_{t}^{2}, \ldots$ be independent $\mathbb{C}$-valued Brownian motions started, respectively, from $z_{1}, z_{2}, \ldots$ under the probability measure $\mathbf{P}^{z_{1}, z_{2}, \ldots}$. We write

$$
B_{t}^{j}=X_{t}^{j}+i Y_{t}^{j}
$$

where $X_{t}^{j}, Y_{t}^{j}$ are independent, one-dimensional Brownian motions. We also sometimes write $B^{j}(t), X^{j}(t), Y^{j}(t)$ for $B_{t}^{j}, X_{t}^{j}, Y_{t}^{j}$. We let

$$
T_{n}^{j}=T_{n}\left(X^{j}\right):=\inf \left\{t>0: X_{t}^{j}=n\right\} \text {. }
$$

Suppose $\bar{p}=\left(p_{1}, \ldots, p_{k}\right)$ is a $k$-tuple $(k \geq 2)$ of positive integers and let

$$
K=K(\bar{p})=p_{1}+\cdots+p_{k} .
$$

We group the Brownian motions into "packets" of size $p_{1}, \ldots, p_{k}$. Let $h(j)=$ $h(j, \bar{p})$ be the packet in which $B^{j}$ is included, that is,

$$
h(j)=l \text { if and only if } p_{1}+\cdots+p_{l-1}+1 \leq j \leq p_{1}+\cdots+p_{l} \text {. }
$$

Let

$$
\Lambda_{n}^{l}=\bigcup_{\{j: h(j)=l\}} B^{j}\left[0, T_{n}^{j}\right]
$$

where we write

$$
B^{j}\left[t_{1}, t_{2}\right]=\left\{B_{t}^{j}: t_{1} \leq t \leq t_{2}\right\}
$$

and let

$$
\bar{\Lambda}_{n}^{l}=\left\{e^{i z}: z \in \Lambda_{n}^{l}\right\} .
$$

Let $\bar{D}_{n}=\bar{D}_{n}(\bar{p})$ be the event

$$
\bar{D}_{n}=\left\{\bar{\Lambda}_{n}^{l} \cap \bar{\Lambda}_{n}^{m}=\varnothing, 1 \leq l<m \leq k\right\} .
$$

Let $F_{n}=F_{n}(\bar{p})$ be the event that for any $m \in[0, n]$, there exists $\theta_{1}, \ldots, \theta_{K}$ such that:

1. $\theta_{j}-Y^{j}\left(T_{m}^{j}\right) \in 2 \pi \mathbb{Z}$.

2. For all $j_{1}, j_{2}$ such that $h\left(j_{1}\right)<h\left(j_{2}\right)$, one has $\theta_{j_{1}}<\theta_{j_{2}}<\theta_{j_{1}}+2 \pi$.

The event $F_{n}$ in some sense implies that the packets of Brownian motions remain in a prescribed order. Let

$$
q_{n}=q_{n}(\bar{p})=\sup \mathbf{P}^{i y_{1}, \ldots, i y_{K}}\left(\bar{D}_{n} \cap F_{n}\right),
$$

where the supremum is over all

$$
0<y_{1}, \ldots, y_{K}<2 \pi
$$

satisfying

$$
y_{j_{1}}<y_{j_{2}} \text { if } h\left(j_{1}\right)<h\left(j_{2}\right)
$$


Note that if we replace (11) by the condition

$$
\left|y_{j_{2}}-y_{j_{1}}\right|<2 \pi, \quad j_{1}, j_{2} \in\{1, \ldots, K\},
$$

we get the same $q_{n}$. From this and the strong Markov property, we get that the $q_{n}$ are submultiplicative, that is,

$$
q_{n+m} \leq q_{n} q_{m}
$$

and hence by standard arguments, if we define

$$
\xi=\xi(\bar{p})=\sup _{n>0} \frac{-\log q_{n}}{n}
$$

(it is easy to check that $\xi<\infty$ using (8); see, e.g., [35]), we get that

$$
q_{n} \approx e^{-n \xi}, \quad n \rightarrow \infty \text {. }
$$

Moreover, the definition of $\xi$ implies that $q_{n} \geq e^{-n \xi}$. We call $\xi(\bar{p})=$ $\xi\left(p_{1}, \ldots, p_{k}\right)$ the $\left(p_{1}, \ldots, p_{k}\right)$ intersection exponent.

In fact, it can be shown (see, e.g., [19]), that there is a $C=C(K)<\infty$ such that for all $n \geq 1$,

$$
q_{n} \leq C e^{-\xi n},
$$

but we will not need this stronger result.

Let

$$
q_{n}^{*}=q_{n}^{*}(\bar{p})=\mathbf{P}^{*}\left(\bar{D}_{n} \cap F_{n}\right)
$$

where the law $\mathbf{P}^{*}$ means that the initial distribution on $\left(i y_{1}, \ldots, i y_{K}\right)$ is the uniform distribution on $[0,2 \pi i]^{K}$ [so that $\exp \left(B^{j}\right)$ are started uniformly on the unit circle]. We will use this notation throughout the paper.

Clearly,

$$
q_{n}^{*} \leq q_{n}
$$

as $q_{n}$ corresponds to the sup over all possible starting points. Define for any $j \leq K$,

$$
u_{j}=B^{j}\left(T_{1}^{j}\right)-1
$$

It is easy to check that the density of the law of $\exp \left(u_{j}\right)$ (with respect to the Lebesgue measure on the circle $\{|z|=1\}$ ) is uniformly bounded. Define for any $t \geq 0$,

$$
B^{j, *}(t)=B^{j}\left(T_{1}^{j}+t\right)-1
$$

$B^{j, *}$ is a Brownian motion started on the imaginary line and the density of $\exp \left(B^{j, *}(0)\right)$ is uniformly bounded (with respect to the Lebesgue measure on the unit circle). This leads readily to the fact that there exists $C=C(K)$ such that

$$
q_{n} \leq C q_{n-1}^{*} .
$$

Combining this with (13) shows that

$$
q_{n}^{*} \approx e^{-n \xi}, \quad n \rightarrow \infty .
$$


2.3. Intersection exponents in strips and half spaces. We will also need to consider the intersection exponent for Brownian motions restricted to stay in the strip

$$
\mathscr{T}=\mathbb{R} \times(0, \pi)=\{x+i y: 0<y<\pi, x \in \mathbb{R}\} .
$$

Let $J_{n}=J_{n}(K)$ be the event

$$
J_{n}=\left\{B^{j}\left[0, T_{n}^{j}\right] \subset \mathscr{T}, \quad j=1, \ldots, K\right\} .
$$

Let $\bar{p}=\left(p_{1}, \ldots, p_{k}\right)$ and $\Lambda_{n}^{l}$ be as above, and let $D_{n}=D_{n}(\bar{p})$ be the event

$$
D_{n}=\left\{\Lambda_{n}^{l} \cap \Lambda_{n}^{m}=\varnothing, 1 \leq l<m \leq k\right\} .
$$

Note that $J_{n} \cap \bar{D}_{n}=J_{n} \cap D_{n}$. Let $F_{n}$ be as above. Note that in this case $D_{n} \cap J_{n} \cap F_{n}=D_{n} \cap J_{n} \cap\left(\cap_{m \in[0, n]} H_{m}\right)$ where

$$
H_{m}=\left\{\forall j_{1}, j_{2} \text { such that } h\left(j_{1}\right)<h\left(j_{2}\right) \text {, one has } Y^{j_{1}}\left(T_{m}^{j_{1}}\right)<Y^{j_{2}}\left(T_{m}^{j_{2}}\right)\right\} \text {. }
$$

Let

$$
\tilde{q}_{n}=\tilde{q}_{n}(\bar{p})=\sup \mathbf{P}^{i y_{1}, \ldots, i y_{K}}\left(J_{n} \cap D_{n} \cap F_{n}\right),
$$

where the supremum is over all

$$
0<y_{1}, \ldots, y_{K}<\pi
$$

satisfying

$$
y_{j_{1}}<y_{j_{2}} \text { if } h\left(j_{1}\right)<h\left(j_{2}\right) .
$$

Again, submultiplicativity shows that there exists a $\tilde{\xi}=\tilde{\xi}(\bar{p})$ such that

$$
\tilde{q}_{n} \approx e^{-n \tilde{\xi}}, \quad n \rightarrow \infty \text {. }
$$

Moreover, $\tilde{q}_{n} \geq e^{-n \tilde{\xi}}$. Again, we could show that $\tilde{q}_{n} \leq C(K) e^{-n \tilde{\xi}}$, but we will not need this. We call $\tilde{\xi}$ the $\left(p_{1}, \ldots, p_{k}\right)$ intersection exponent for Brownian motions restricted to a strip. We will also refer to $\tilde{\xi}$ as the intersection exponent for Brownian motions restricted to a half space, since that is what the paths look like under conformal transformation by the exponential map. If $k=1$, we set

$$
\tilde{\xi}(p)=p .
$$

This is the natural definition, since in this case $J_{n} \cap D_{n} \cap F_{n}=J_{n}$, and (8) gives

$$
\sup _{y \in[0, \pi]} \mathbf{P}^{i y}\left(J_{n}\right)=\mathbf{P}^{i \pi / 2}\left(J_{n}\right) \approx e^{-p n}, \quad n \rightarrow \infty .
$$

We define

$$
\tilde{q}_{n}^{*}=\tilde{q}_{n}^{*}(\bar{p})=\mathbf{P}^{*}\left(J_{n} \cap D_{n} \cap F_{n}\right),
$$

(the initial distribution is the uniform distribution on $[0,2 \pi i]^{K}$ ). Note that the event in the last formula can hold only if the initial points satisfy (15) and (16). As before, we can see that for some $C=C(K)<\infty$,

$$
\tilde{q}_{n}^{*} \leq \tilde{q}_{n} \leq C \tilde{q}_{n-1}^{*} .
$$


In particular,

$$
\tilde{q}_{n}^{*} \approx e^{-n \tilde{\xi}}, \quad n \rightarrow \infty .
$$

It is sometimes more convenient to use the "conditioned half-space" exponent

$$
\hat{\xi}(\bar{p})=\tilde{\xi}(\bar{p})-\left(p_{1}+\cdots+p_{k}\right) .
$$

We shall see that this is the exponent for Brownian motions conditioned to be in the strip, that is,

$$
\mathbf{P}^{*}\left(J_{n} \cap D_{n} \cap F_{n} \mid J_{n}\right) \approx \exp (-n \hat{\xi}(\bar{p})), \quad n \rightarrow \infty .
$$

2.4. Conditioned Brownian motions and exponents. Let $\hat{X}_{t}^{1}, \hat{X}_{t}^{2}, \ldots$ be independent three-dimensional Bessel processes and let

$$
\hat{T}_{n}^{j}=T_{n}\left(\hat{X}^{j}\right)=\inf \left\{t>0: \hat{X}_{t}^{j}=n\right\} .
$$

Let $Y^{1}, Y^{2}, \ldots$ be independent one-dimensional Brownian motions, independent of $\hat{X}_{t}^{1}, \hat{X}_{t}^{2}, \ldots$, and define the processes $W^{1}, W^{2}, \ldots$ by

$$
W_{t}^{j}=\hat{X}_{t}^{j}+i Y_{t}^{j} .
$$

The processes $W^{1}, W^{2}, \ldots$ can be viewed as independent two-dimensional Brownian motions conditioned to remain forever in the half-plane $\{z: \mathfrak{i}(z)>$ $0\}$. In the rest of this paper we will simply refer to such processes as "conditioned Brownian motions" (or CBMs in short).

Let $\bar{p}=\left(p_{1}, \ldots, p_{k}\right)$ be a $k$-tuple of positive integers and define $K$ and $h(j)$ as above. Let

$$
\Theta_{n}^{l}=\bigcup_{j: h(j)=l} W^{j}\left[0, \hat{T}_{n}^{j}\right]
$$

and

$$
\bar{\Theta}_{n}^{l}=\left\{\exp (z): z \in \Theta_{n}^{l}\right\}
$$

Let $\bar{E}_{n}=\bar{E}_{n}(\bar{p})$ be the event,

$$
\bar{E}_{n}=\left\{\bar{\Theta}_{n}^{l} \cap \bar{\Theta}_{n}^{m}=\varnothing, 1 \leq l<m \leq k\right\}
$$

and $\hat{F}_{n}=\hat{F}_{n}(\bar{p})$ the event defined analogously to $F_{n}$ (replacing the Brownian motions $B^{j}$ 's by the CBM $W^{j}$ 's). Note that by topological considerations,

Let

$$
\bar{E}_{n} \cap \hat{F}_{n}=\bar{E}_{n} \cap \hat{F}_{0} .
$$

$$
r_{n}^{*}=r_{n}^{*}(\bar{p})=\mathbf{P}^{*}\left(\bar{E}_{n} \cap \hat{F}_{n}\right),
$$

(the initial distribution of $\bar{z}$ is again the uniform distribution on $[0,2 i \pi]^{K}$ ).

We can bound $q_{n}^{*}(\bar{p})$ in terms of $r_{n}^{*}(\bar{p})$ because the path of a conditioned Brownian motion can be viewed as a subpath of the path of a Brownian motion: Suppose that $B^{j}$ is a planar Brownian motion started with the 
uniform distribution on $[0,2 \pi i]$. Define

and for all $t \geq 0$,

$$
\tau_{0, n}^{j}=\sup \left\{t<T_{n}^{j}: X_{t}^{j}=0\right\}
$$

$$
B^{j, \#}(t)=B^{j}\left(\tau_{0, n}^{j}+t\right)
$$

It follows from (9) that the law of $\exp \left(B^{j, \#}\right)$ up to its hitting time of the circle of radius $\log n$ is exactly that of $\exp \left(W^{j}(t)\right)$ up to its hitting time of the circle of radius $\log n$ (in both cases, the starting points are chosen uniformly on the unit circle). Hence we immediately get that for any $n>0$,

$$
q_{n}^{*}(\bar{p}) \leq r_{n}^{*}(\bar{p})
$$

The intersection exponent for conditioned Brownian motions can be defined in a similar way as the intersection exponent for Brownian motion, but as the next proposition shows, the exponents are the same.

Proposition 1.

$$
r_{n}^{*}(\bar{p}) \approx \exp (-n \xi(\bar{p})), \quad n \rightarrow \infty .
$$

Proof. Since $r_{n}^{*}(\bar{p}) \geq q_{n}^{*}(\bar{p})$, we need only find an upper bound on $r_{n}^{*}(\bar{p})$. Choose $\bar{z}$, and write $\mathbf{P}$ and $\mathbf{P}^{\bar{z}}$. Let

$$
\begin{array}{r}
\bar{E}_{n}^{\prime}=\left\{\exp \left(W^{j_{1}}\left[\hat{T}_{1}^{j_{1}}, \hat{T}_{n}^{j_{1}}\right]\right)\right. \\
\left.1 \leq j_{1}, j_{2} \leq K, h\left(j_{1}\right) \neq h\left(j_{2}\right)\right\} .
\end{array}
$$

Then

and hence

$$
\hat{F}_{n} \cap \bar{E}_{n} \subset \hat{F}_{n} \cap \bar{E}_{n}^{\prime}
$$

$$
\mathbf{P}\left(\hat{F}_{n} \cap \bar{E}_{n}\right) \leq \mathbf{P}\left(\hat{F}_{n} \cap \bar{E}_{n}^{\prime}\right) \leq \mathbf{P}\left(\hat{F}_{n} \cap \bar{E}_{n}^{\prime} \mid \hat{F}_{1}\right) .
$$

The probability on the right is with respect to CBMs and depends only on their values after they first hit the line $\mathfrak{R}(z)=1$, but this is the same as the probability for Brownian motions conditioned so that they stay in $\{\Re(z)>0\}$. Since the probability that $K$ independent Brownian motions starting at $\{\Re(z)=1\}$ reach $\{\Re(z)=n\}$ without hitting $\{\Re(z)=0\}$ is $n^{-K}$, we get easily that

$$
\mathbf{P}\left(\hat{F}_{n} \cap \bar{E}_{n}^{\prime} \mid \hat{F}_{1}\right) \leq n^{K} q_{n-1}(\bar{p}) \approx e^{-n \xi(\bar{p})}, \quad n \rightarrow \infty
$$

and this concludes the proof of the proposition.

We can do similarly for exponents in strips. Let $\hat{J}_{n}=\hat{J}_{n}(K)$ be the event

$$
\hat{J}_{n}=\left\{W^{j}\left[0, \hat{T}_{n}^{j}\right] \subset \mathscr{T}, j=1, \ldots, K\right\},
$$

let $E_{n}=E_{n}(\bar{p})$ be the event

$$
E_{n}=\left\{\Theta_{n}^{l} \cap \Theta_{n}^{m}=\varnothing, 1 \leq l<m \leq k\right\}
$$


and put

Let

$$
\hat{E}_{n}=E_{n} \cap \hat{J}_{n}
$$

$$
\tilde{r}_{n}^{*}=\tilde{r}_{n}^{*}(\bar{p})=\mathbf{P} *\left(\hat{E}_{n} \cap \hat{F}_{0}\right)=\mathbf{P}^{*}\left(\hat{E}_{n} \cap \hat{F}_{n}\right) .
$$

Then we can prove the following in the same way as Proposition 1.

Proposition 2.

$$
\tilde{r}_{n}^{*}(\bar{p}) \approx \exp (-n \tilde{\xi}(\bar{p})), \quad n \rightarrow \infty
$$

Note that in particular, when $p=(1)$, this shows that

$$
\mathbf{P}^{*}\left(W^{1}\left[0, \hat{T}_{n}^{1}\right] \subset \mathscr{T}\right) \approx e^{-n}
$$

as $\tilde{\xi}(1)=1$ (actually, this could have been easily derived directly).

2.5. Generalized exponents I. We are now going to define exponents

and

$$
\tilde{\xi}\left(\lambda_{1}, p_{1}, \lambda_{2}, \ldots, p_{k}, \lambda_{k+1}\right)
$$

$$
\xi\left(\lambda_{1}, p_{1}, \ldots, \lambda_{k}, p_{k}\right)
$$

when $\lambda_{j}$ 's are nonnegative real numbers and $p_{j}$ 's are positive integers.

We start by defining for every $\lambda \geq 0$,

$$
\tilde{\xi}(\lambda)=\lambda \text {. }
$$

This agrees with our previous definition if $\lambda$ is a positive integer.

Now, fix $k \geq 1, \bar{p}=\left(p_{1}, \ldots, p_{k}\right)$, and keep the same notation as in Section 2.4. We let $\bar{\lambda}=\left(\lambda_{1}, \lambda_{2}, \ldots, \lambda_{k+1}\right)$. Recall that for $1 \leq l \leq k$,

$$
\Theta_{n}^{l}=\bigcup_{j: h(j)=l} W^{j}\left[0, \hat{T}_{n}^{j}\right] .
$$

For ease, we set

$$
\begin{aligned}
\Theta_{n}^{0} & =\{x: 0 \leq x \leq n\}, \\
\Theta_{n}^{k+1} & =\{x+i \pi: 0 \leq x \leq n\} .
\end{aligned}
$$

For $2 \leq l \leq k$, let $\hat{F}_{m}^{l}$ be the event

$$
\hat{F}_{m}^{l}=\left\{\forall j, j^{\prime}, \mathscr{J}\left[W^{j}\left(\hat{T}_{m}^{j}\right)\right]<\mathscr{J}\left[W^{j^{\prime}}\left(\hat{T}_{m}^{j^{\prime}}\right)\right] \text { if } h(j)=l-1, h\left(j^{\prime}\right)=l\right\},
$$

and let

$$
\begin{aligned}
\hat{F}_{m}^{1} & =\left\{\mathscr{J}\left[W^{j}\left(\hat{T}_{m}^{j}\right)\right]>0 \text { if } h(j)=1\right\}, \\
\hat{F}_{m}^{k+1} & =\left\{\mathscr{J}\left[W^{j}\left(\hat{T}_{m}^{j}\right)\right]<\pi \text { if } h(j)=k\right\} .
\end{aligned}
$$

Note that $\hat{F}_{0}^{l}$ depends only on the positions of the starting points. 
Let $O_{n}^{l}, l=1, \ldots, k+1$, be the open domain bounded above by $\Theta_{n}^{l}$, below by $\Theta_{n}^{l-1}$, on the left by a subinterval of $\{\Re(z)=0\}$ and on the right by a subinterval of $\{\Re(z)=n\}$. This domain is well defined if the event $\hat{F}_{0}^{l}$ holds and $\Theta_{n}^{l} \cap \Theta_{n}^{l-1}=\varnothing$. If $\hat{F}_{0}^{l}$ does not hold or $\Theta_{n}^{l} \cap \Theta_{n}^{l-1} \neq \varnothing$, we set $O_{n}^{l}=\varnothing$. Note that (at least up to a null event),

$$
\hat{F}_{0} \cap \hat{E}_{n}=\left\{O_{n}^{l} \neq \varnothing, l=1, \ldots, k+1\right\} .
$$

Let $\breve{W}_{t}$ be another independent conditioned Brownian motion started with the uniform distribution on $[0,2 \pi i]$ under the probability measure $\breve{\mathbf{P}}$ and

$$
\breve{T}_{n}=\inf \left\{t>0: \mathfrak{R}\left(\breve{W}_{t}\right)=n\right\} .
$$

Let

$$
Z_{n}^{l}=Z_{n}^{l}\left(O_{n}^{l}\right)=\breve{\mathbf{P}}\left(\breve{W}\left(0, \breve{T_{n}}\right) \subset O_{n}^{l}\right) .
$$

Note that $Z_{n}^{l}$ is a deterministic function of $O_{n}^{l}$. Clearly, $Z_{n}^{l}=0$ if $L_{n}^{l}=\varnothing$.

Let

$$
s_{n}^{*}=s_{n}^{*}(\bar{p}, \bar{\lambda})=\mathbf{E}^{*}\left[\prod_{l=1}^{k+1}\left(Z_{n}^{l}\right)^{\lambda_{l}}\right] .
$$

If $\lambda_{l}=0$, we use the convention $0^{0}=0$, that is, $\left(Z_{n}^{l}\right)^{0}$ is the indicator function of the event $\left\{Z_{n}^{l}>0\right\}$. Using the decomposition of conditioned Brownian motions [see (10)], it is not difficult to check that there is a constant $c$ such that

$$
s_{m+n+1}^{*} \leq c s_{m}^{*} s_{n}^{*}
$$

and therefore $u_{n}=c s_{n-1}^{*}$ is submultiplicative. We define

$$
\tilde{\xi}=\tilde{\xi}\left(\lambda_{1}, p_{1}, \ldots, p_{k}, \lambda_{k+1}\right)=\sup _{n>0} \frac{-\log u_{n}}{n} .
$$

It is easy using (17) to see that $\tilde{\xi}<\infty$. For instance, when $k=1, \bar{p}=(1)$ and $\lambda \in(0, \infty)$, we get that

$$
\begin{aligned}
& s_{n}^{*}((1),(\lambda, 0)) \\
& \quad \leq \mathbf{E}^{*}\left[1_{\left\{W^{1}\left[0, \hat{T}_{n}^{1}\right] \subset[0, n] \times(0, \pi(1-\eta))\right\}} \breve{\mathbf{P}}\left(\breve{W}\left[0, \breve{T}_{n}\right] \subset[0, n] \times(\pi(1-\eta), \pi)\right)^{\lambda}\right] \\
& \quad \approx \exp \left(-\frac{n}{1-\eta}\right) \exp \left(-\frac{n \lambda}{\eta}\right) \quad \text { when } n \rightarrow \infty,
\end{aligned}
$$

so that [this corresponds to the choice $\eta=\sqrt{\lambda} /(1+\sqrt{\lambda})$ ]

$$
\tilde{\xi}(0,1, \lambda) \leq(1+\sqrt{\lambda})^{2} .
$$

In the rest of the paper, we will use the notation $\tilde{\xi}(1, \lambda)=\tilde{\xi}(0,1, \lambda)$.

Similarly, one can see that

$$
\tilde{\xi}\left(\lambda_{1}, p_{1}, \ldots, p_{k}, \lambda_{k+1}\right) \leq\left(\sum_{j=1}^{k} \sqrt{p_{j}}+\sum_{j=1}^{k+1} \sqrt{\lambda_{j}}\right)^{2}
$$


by dividing the rectangle $[0, n] \times[0, \pi]$ into $2 k+1$ well-chosen rectangles (see, e.g., [35]).

Hence,

when $n \rightarrow \infty$.

$$
s_{n}^{*} \approx u_{n} \approx \exp (-n \tilde{\xi})
$$

It is easy to check that if $\lambda_{1}, \ldots, \lambda_{k+1}$ are positive integers, then this extends our previous definition of $\tilde{\xi}$.

We now briefly focus on some simple properties of the mapping $\bar{\lambda} \rightarrow \tilde{\xi}(\bar{p}, \bar{\lambda})$.

Suppose now that $\bar{p}$ is fixed and that $\bar{\lambda}$ and $\bar{\mu}=\left(\mu_{1}, \ldots, \mu_{k+1}\right)$ are in $\mathbb{R}_{+}^{k+1}$. Schwarz's inequality yields

$$
\begin{aligned}
s_{n}^{*}\left(\bar{p}, \frac{\bar{\lambda}+\bar{\mu}}{2}\right) & =\mathbf{E}^{*}\left[\prod_{l=1}^{k+1}\left(Z_{n}^{l}\right)^{\left(\lambda_{l}+\mu_{l}\right) / 2}\right] \\
& \leq \mathbf{E}^{*}\left[\prod_{l=1}^{k+1}\left(Z_{n}^{l}\right)^{\lambda_{l}}\right]^{1 / 2} \mathbf{E}^{*}\left[\prod_{l=1}^{k+1}\left(Z_{n}^{l}\right)^{\mu_{l}}\right]^{1 / 2} \\
& =\left(s_{n}^{*}(\bar{p}, \bar{\lambda}) s_{n}^{*}(\bar{p}, \bar{\mu})\right)^{1 / 2} .
\end{aligned}
$$

This shows that the function $\bar{\lambda} \mapsto-\log s_{n}^{*}(\bar{p}, \bar{\lambda})$ is concave so that

$$
\bar{\lambda} \mapsto \tilde{\xi}\left(\lambda_{1}, p_{1}, \lambda_{2}, p_{2}, \ldots, p_{k}, \lambda_{k+1}\right)
$$

is also concave. In particular, it is continuous on $(0, \infty)^{k+1}$.

As almost surely,

$$
Z_{n}^{j} \leq \breve{\mathbf{P}}\left(\breve{W}\left(0, \breve{T}_{n}\right) \subset \mathscr{T}\right) \approx \exp (-n) \text { when } n \rightarrow \infty,
$$

one immediately sees that

$$
\lambda_{j} \mapsto \tilde{\xi}\left(\lambda_{1}, p_{1}, \ldots, p_{k}, \lambda_{k+1}\right)
$$

is strictly increasing and that for any $\lambda, \lambda^{\prime} \geq 0$,

$$
\tilde{\xi}\left(\lambda^{\prime}, 1, \lambda\right) \geq \lambda+\tilde{\xi}\left(\lambda^{\prime}, 1\right) .
$$

In particular, we see that [combining this with (18)]

$$
\tilde{\xi}(1, \lambda) \sim \lambda, \quad \lambda \rightarrow \infty .
$$

Define for all $\lambda \geq 0$,

$$
\tilde{\kappa}(\lambda)=\tilde{\xi}(1, \lambda) .
$$

We have just seen that $\tilde{\kappa}$ is strictly increasing, that $\lim _{+\infty} \tilde{\kappa}=+\infty$ and that it is continuous on $(0, \infty)$. Continuity at 0 follows from (18). Hence, $\tilde{\kappa}$ is bijective from $[0, \infty)$ into $[1, \infty)$.

An argument similar to that used to derive (18) shows that for any $\lambda, \lambda^{\prime} \geq 0$,

$$
\tilde{\xi}\left(\lambda^{\prime}, 1, \lambda\right) \leq\left(\sqrt{\tilde{\xi}\left(\lambda^{\prime}, 1\right)}+\sqrt{\lambda}\right)^{2}
$$


This implies easily that the (concave) function

$$
\left(\lambda, \lambda^{\prime}\right) \mapsto \tilde{\xi}\left(\lambda^{\prime}, 1, \lambda\right)
$$

is in fact continuous on $[0, \infty)^{2}$.

Note that symmetry implies

$$
\tilde{\xi}\left(\lambda_{1}, p_{1}, \ldots, p_{k}, \lambda_{k+1}\right)=\tilde{\xi}\left(\lambda_{k+1}, p_{k}, \ldots, p_{1}, \lambda_{1}\right) .
$$

We also define

$$
\begin{gathered}
\tilde{\xi}\left(\lambda_{1}, p_{1}, \ldots, \lambda_{l-1}, p_{l}, p_{l+1}, \lambda_{l+1}, \ldots, p_{k}, \lambda_{k+1}\right) \\
=\tilde{\xi}\left(\lambda_{1}, p_{1}, \ldots, p_{l}, 0, p_{l+1}, \ldots, p_{k}, \lambda_{k+1}\right) .
\end{gathered}
$$

By continuing we can define $\tilde{\xi}$ for any $k$-tuple of nonnegative real numbers such that any pair of consecutive numbers (in the $k$-tuple) contains at least one positive integer. We also extend the definition of the conditioned half-space exponents in the natural way,

$$
\begin{aligned}
\hat{\xi}\left(\lambda, p_{1}, \ldots, p_{k}, \lambda_{k+1}\right)= & \tilde{\xi}\left(\lambda_{1}, p_{1}, \ldots, p_{k}, \lambda_{k+1}\right) \\
& -\left(\lambda_{1}+p_{1}+\cdots+p_{k}+\lambda_{k+1}\right) .
\end{aligned}
$$

The exponents $\xi\left(\lambda_{1}, p_{1}, \ldots, \lambda_{k}, p_{k}\right)$ are defined similarly. Suppose first that $k \geq 2$. Let $\Theta_{n}^{1}, \ldots, \Theta_{n}^{k}$ be as above. Define $\Theta_{n}^{0}=\Theta_{n}^{k}$. When $\bar{E}_{n} \cap \hat{F}_{n}$ is satisfied, let $U_{n}^{l}, l=1, \ldots, k$ denote the subset of the annulus $\{z: 1<|z|<$ $\exp n\}$ bounded by $\{|z|=1\}, \exp \left(\Theta_{n}^{l-1}\right),\{|z|=\exp n\}$ and $\exp \left(\Theta_{n}^{l}\right)$ (note that there are only $k$ domains $U_{n}^{1}, \ldots, U_{n}^{k}$ here).

The definition of the random variables $Z_{n}^{l}$ are modified as follows:

$$
Z_{n}^{l}=\breve{\mathbf{P}}\left(\exp \left(\breve{W}\left(0, \breve{T}_{n}\right)\right) \subset U_{n}^{l}\right) .
$$

We set

$$
s_{n}^{\prime}=s_{n}^{\prime}(\bar{p}, \bar{\lambda}):=\mathbf{E}^{*}\left[1_{\left\{\bar{E}_{n} \cap \hat{F}_{n}\right\}} \prod_{l=1}^{k}\left(Z_{n}^{l}\right)^{\lambda_{l}}\right] .
$$

Again it is easy to check that this extends our previous definition when all of the $\lambda_{l}$ 's are positive integers.

We will also write

$$
\xi\left(\lambda_{1}, p_{1}, \ldots, \lambda_{k}, p_{k}\right)=\xi\left(p_{k}, \lambda_{k}, \ldots, p_{1}, \lambda_{1}\right) .
$$

Note that by symmetry, for each $2 \leq l \leq k$,

$$
\xi\left(\lambda_{l}, p_{l}, \ldots, \lambda_{k}, p_{k}, \lambda_{1}, p_{1}, \ldots, \lambda_{l-1}, p_{l-1}\right)=\xi\left(\lambda_{1}, p_{1}, \ldots, \lambda_{k}, p_{k}\right) .
$$

When $k=1$, one has to clarify the definition of $U_{n}^{1}$. There could be several choices of connected components (if some of the $p_{1}$ conditioned Brownian motions do not intersect). If such is the case, then define $U_{n}^{1}$ as the connected component that maximizes

$$
\breve{\mathbf{P}}\left(\exp \left(\breve{W}\left(0, \breve{T}_{n}\right)\right) \subset U_{n}^{1}=\varnothing\right) .
$$


One can then check that this leads to the definition of $\xi\left(\lambda_{1}, n_{1}\right)$ with

$$
\mathbf{E}^{*}\left[\left(Z_{n}^{1}\right)^{\lambda_{1}}\right] \approx \exp \left[-n \xi\left(\lambda_{1}, n_{1}\right)\right] .
$$

We safely leave this to the reader. Note that $\xi(p, 0)$ is the so-called disconnection exponent for $p$ planar Brownian paths (see, e.g. [33]) that measures the decay of the probability that the union of $p$ independent Brownian paths do not disconnect a point that is close to their starting points. More precisely, with the previous notations,

$$
\mathbf{P}^{*}\left[\exp \left(\Theta_{n}^{1}\right) \text { does not disconnect } 0 \text { from infinity }\right] \approx \exp (-n \xi(p, 0)) \text {. }
$$

Again, Schwarz's inequality shows that $\bar{\lambda} \mapsto \xi(\bar{p}, \bar{\lambda})$ is a concave function. In particular, $\lambda \mapsto \xi(1, \lambda)$ is continuous on $(0, \infty)$.

\section{Conformal invariance.}

3.1. Extremal distance. We first recall some well-known facts concerning conformal invariance. Let $\mathscr{D}$ be a bounded, simply connected domain in $\mathbb{C}$ whose boundary $\partial \mathscr{D}$ is a closed Jordan curve. Let $\partial_{1}, \partial_{2} \subset \partial \mathscr{D}$ be disjoint, connected, closed sets that are not singletons and let $\partial_{3}$ and $\partial_{4}$ denote the two connected components of $\partial \mathscr{D} \backslash\left(\partial_{1} \cup \partial_{2}\right)$ defined in such a way that $\partial_{1}$, $\partial_{3}, \partial_{2}$ and $\partial_{4}$ are ordered counterclockwise. The domains we will need to consider in this paper are particularly simple; these domains will satisfy

where

$$
\begin{gathered}
\partial_{1}=\left\{i y: s_{1} \leq y \leq t_{1}\right\}, \\
\partial_{2}=\left\{n+i y: s_{2} \leq y \leq t_{2}\right\}, \\
\partial \mathscr{D} \backslash\left(\partial_{1} \cup \partial_{2}\right) \subset\{0<\mathfrak{R}(z)<n\},
\end{gathered}
$$

$$
\begin{gathered}
n>0, \\
0 \leq s_{1}<t_{1} \leq 2 \pi, \\
0<t_{2}-s_{2}<2 \pi .
\end{gathered}
$$

Moreover, for every $m$, the cross section

$$
\mathscr{D} \cap\{\Re(z)=m\} \text {, }
$$

will be contained in an interval of length $2 \pi$. We will call such domains path domains of length $n$. In this case $\partial_{3}$ is the "lower" boundary and $\partial_{4}$ is the "upper" boundary. Examples of such domains are the sets $O_{n}^{l}$ from Section 2.5 .

Let $\mathscr{T}_{n}$ be the $n \times \pi$ rectangle

$$
\mathscr{T}_{n}=\{z \in \mathbb{C}: 0<\mathfrak{R}(z)<n, 0<\mathfrak{I}(z)<\pi\},
$$

whose boundary is the union of

$$
\begin{aligned}
& \partial_{1}^{\prime}=\{i y: 0 \leq y \leq \pi\}, \\
& \partial_{2}^{\prime}=\partial_{2, n}^{\prime}=\{n+i y: 0 \leq y \leq \pi\}, \\
& \partial_{3}^{\prime}=\partial_{3, n}^{\prime}=\{x: 0<x<n\}, \\
& \partial_{4}^{\prime}=\partial_{4, n}^{\prime}=\{x+i \pi: 0<x<n\} .
\end{aligned}
$$


There exist a unique $L=L(\mathscr{D})=L\left(\mathscr{D}, \partial_{1}, \partial_{2}\right)$ and a unique conformal transformation

$$
f: \mathscr{D} \rightarrow \mathscr{T}_{L},
$$

such that $f$ can be extended to a continuous function

$$
f: \overline{\mathscr{D}} \rightarrow \overline{\mathscr{T}}_{L},
$$

with

$$
f\left(\partial_{j}\right)=\partial_{j}^{\prime}, \quad j=1,2,3,4 .
$$

$L / \pi$ is called the extremal distance between $\partial_{1}$ and $\partial_{2}$ in $\mathscr{D}$. As we will make an extensive use of this notion, let us first recall some basic facts (see, e.g., [1] for more information).

The extremal distance can be defined alternatively as follows (see [1], Definition 4.1):

$$
\frac{L(\mathscr{D})}{\pi}=\sup _{\rho} \frac{\inf _{\gamma}\left(\operatorname{length}_{\rho}(\gamma)\right)^{2}}{\operatorname{area}_{\rho}(\mathscr{D})},
$$

where the sup is over all $C^{\infty}$ metrics on $\mathscr{D}$, and the infimum is over all smooth paths $\gamma$ joining $\partial_{1}$ to $\partial_{2}$ in $\mathscr{D}$ (length and area $_{\rho}$ denote respectively the length and the area with respect to the metric $\rho$ ).

In particular, when $\mathscr{D}$ is a path domain of length $n$ as above, the Euclidean distance between any path joining $\partial_{1}$ to $\partial_{2}$ is at least $n$, so that

$$
\frac{L(\mathscr{D})}{\pi} \geq \frac{n^{2}}{\operatorname{area}(\mathscr{D})},
$$

where "area" from now on denotes the Euclidean area. Suppose now that $\mathscr{D}^{1}, \ldots, \mathscr{D}^{k}$ are $k$ disjoint path domains of length $n$ that are subsets of $\mathscr{T}_{n}$, and that $L\left(\mathscr{D}^{j}\right.$ ) (for each $j=1, \ldots, k$ ) denotes $\pi$ times the extremal distance between two parts of the boundary of $\mathscr{D}^{j}$ that are respectively subsets of $\{z$ : $\mathfrak{i}(z)=0\}$ and $\{z: \mathfrak{i}(z)=n\}$. Then the previous remark shows that for all positive numbers $\alpha_{1}, \ldots, \alpha_{k}$,

$$
\sum_{j=1}^{k} \alpha_{j} L\left(\mathscr{D}^{j}\right) \geq \pi n^{2} \sum_{j=1}^{k} \frac{\alpha_{j}}{\operatorname{area}\left(\mathscr{D}^{j}\right)} .
$$

However, as the sets $\mathscr{D}^{j}$ are disjoint subsets of $\mathscr{T}_{n}$,

$$
\sum_{j=1}^{k} \operatorname{area}\left(\mathscr{D}^{j}\right) \leq n \pi .
$$

By maximizing the right-hand side of (22) subject to the constraint (23) yields the lower bound in the following lemma. 
Lemma 3. Suppose $\mathscr{D}^{1}, \ldots, \mathscr{D}^{k}$ are disjoint path domains of length $n$ that are subsets of $\mathscr{T}_{n}$. Then for all nonnegative $\alpha_{1}, \ldots, \alpha_{k}$,

$$
\sum_{j=1}^{k} \alpha_{j} L\left(\mathscr{D}^{j}\right) \geq n\left(\sum_{j=1}^{k} \sqrt{\alpha_{j}}\right)^{2} .
$$
by

If $\mathscr{D}$ is a path domain of length $n$ as above, define its symmetrization $s \mathscr{D}$

$$
s \mathscr{D}=\{x+i y:|x|+i y \in \mathscr{D}\} \cup\left\{i y: s_{1}<y<t_{1}\right\} .
$$

Note that there exists a unique $L^{\prime}>0$ and a unique conformal map $\tilde{f}$ that maps $s \mathscr{D}$ onto $s \mathscr{T}_{L^{\prime}}$ in such a way that the images of the four "corners" $n+i s_{2}, n+i t_{2},-n+i t_{2}$ and $-n+i s_{2}$ are the four corners $L^{\prime}, L^{\prime}+i \pi$, $-L^{\prime}+i \pi$ and $-L^{\prime}$. Because of the uniqueness of this conformal map $\tilde{f}$, it is clear $\tilde{f}$ is "symmetric" and in particular that $\tilde{f}\left(\partial_{1}\right)=(0, i \pi)$. Hence, $\tilde{f}$ maps conformally $\mathscr{D}$ onto $\mathscr{T}_{L^{\prime}}$ so that in fact $L=L^{\prime}$ and $\tilde{f}=f$ on $\mathscr{D}$. In particular, this shows that $f$ is in fact analytic on $\left(i s_{1}, i t_{1}\right)$ and that for all $z \in$ $\left(i s_{1}, i t_{1}\right), f^{\prime}(z)$ is a nonnegative real number. For $s_{1}<y<t_{1}$, let

$$
g(y)=f^{\prime}(i y) \text {. }
$$

Then

$$
g(y)=\left.\frac{\partial(\Re f)}{\partial(\mathfrak{R}(z))}\right|_{z=i y}=\left.\frac{\partial(\mathscr{J} f)}{\partial(\mathscr{J}(z))}\right|_{z=i y}
$$

3.2. Conformally invariant path measure. In this section we describe a conformally invariant measure on paths in a path domain $\mathscr{D}$. Let us stress that this will be a positive measure but not necessarily a probability measure.

Suppose that $\mathscr{D}$ denotes a path domain of length $n$ as in the previous section and define $\partial_{1}, \partial_{2}, f, g, L=L(\mathscr{D}), \mathscr{T}_{L}, \partial_{1}^{\prime}=\partial_{1, L}$, and so on, as in the previous section. Suppose that $B$ and $W$ denote respectively a Brownian motion and a CBM started from $z \in \mathbb{C}$ under the probability measure $\mathbf{P}^{z}$. Let $\tau=\tau(\mathscr{D})$ and $\tau^{\prime}=\tau\left(\mathscr{T}_{L}\right)$ be the exit times of $B$ from the respective domains. Then, if $g$ is as defined in the previous section,

$$
\begin{aligned}
\mathbf{P}^{i y}\left(W\left(0, \hat{T}_{n}\right) \subset \mathscr{D}\right) & =\lim _{\varepsilon \rightarrow 0} \mathbf{P}^{i y+\varepsilon}\left(W\left(0, \hat{T}_{n}\right) \subset \mathscr{D}\right) \\
& =\lim _{\varepsilon \rightarrow 0}(n / \varepsilon) \mathbf{P}^{i y+\varepsilon}\left(B(\tau) \in \partial_{2}\right) \\
& =\lim _{\varepsilon \rightarrow 0}(n / \varepsilon) \mathbf{P}^{f(i y+\varepsilon)}\left(B\left(\tau^{\prime}\right) \in \partial_{2}^{\prime}\right) \\
& =\lim _{\varepsilon \rightarrow 0}(n / \varepsilon) \mathbf{P}^{f(i y)+\varepsilon g(y)}\left(B\left(\tau^{\prime}\right) \in \partial_{2}^{\prime}\right) \\
& =(n g(y) / L) \mathbf{P}^{f(i y)}\left(W\left(0, \hat{T}_{L}\right) \subset \mathscr{T}_{L}\right) .
\end{aligned}
$$


In particular, for all $s_{1}<s<t<t_{1}$,

$$
\begin{aligned}
\frac{1}{n} \int_{s}^{t} \mathbf{P}^{i y}\left(W\left(0, \hat{T}_{n}\right) \subset \mathscr{D}\right) d y & =\frac{1}{L} \int_{s}^{t} \mathbf{P}^{f(i y)}\left(W\left(0, \hat{T}_{L}\right) \subset \mathscr{T}_{L}\right) g(y) d y \\
& =\frac{1}{L} \int_{f(i s) / i}^{f(i t) / i} \mathbf{P}^{i y}\left(W\left(0, \hat{T}_{l}\right) \subset \mathscr{T}_{L}\right) d y .
\end{aligned}
$$

We therefore define the measure $\mu_{\mathscr{g}}$ on processes $\left(W_{t}, t \leq \hat{T}_{n}\right)$ as follows: Define first the probability measure $P^{i y}$ on CBM $\left(W_{t}, t \leq \hat{T}_{n}\right)$ started from $i y$. If $d y$ denotes the Lebesgue measure on $\mathbb{R}$,

$$
\mu_{\mathscr{D}}(\cdot)=\frac{1}{2 \pi n} \int_{\mathbb{R}} d y \mathbf{E}^{i y}\left((\cdot) 1_{W\left(0, \hat{T}_{n}\right) \subset \mathscr{D}}\right) .
$$

In other words, $n \mu_{\mathscr{D}}$ is the same measure as that obtained by starting a CBM $W$ with the uniform measure on $[0,2 \pi i]$; taking the path $W\left[0, \hat{T}_{n}\right]$ and killing all paths with $W\left(0, \hat{T}_{n}\right) \not \subset \mathscr{D}$.

It is obvious from our definition that the measure $\mu_{\mathscr{D}}$ have the following properties:

1. Suppose that $\mathscr{D}^{\prime} \subset \mathscr{D}$ and that $\mathscr{D}^{\prime}$ and $\mathscr{D}$ are two path domains of same length $n$. Then,

$$
\mu_{\mathscr{D}}(\cdot)=\mu_{\mathscr{D}}\left((\cdot) 1_{W\left(0, \hat{T}_{n}\right) \subset \mathscr{D}^{\prime}}\right) .
$$

2. The measure $\mu_{\mathscr{D}}$ is conformally invariant in the following sense: if $\mathscr{D}$ and $\mathscr{D}^{\prime}$ are two path domains of length $n$ and $n^{\prime}$ such that there exists a conformal mapping $f$ from $\mathscr{D}$ onto $\mathscr{D}^{\prime}$ with $f\left(\partial_{1}\right)=\partial_{1}^{\prime}$ and $f\left(\partial_{2}\right)=\partial_{2}^{\prime}$, then the law of the trace

$$
\left\{f\left(W_{t}\right), t \leq \hat{T}_{n}\right\}
$$

under the measure $\mu_{\mathscr{D}}$ is identical to the law of

$$
\left\{W_{t}, t \leq \hat{T}_{n^{\prime}}\right\}
$$

under the measure $\mu_{\mathscr{D}}$.

It suffices to prove this in the case where $\mathscr{D}^{\prime}=\mathscr{T}_{l}$ and this is just a consequence of conformal invariance of planar Brownian motion and of (24).

There are more universal definitions of this conformal invariant measure on Brownian excursions in any simply connected domain, but we will not need them here.

We are now going to derive a first useful consequence of this conformal invariance: the total mass of $n \mu_{\mathscr{D}}$ is the probability that $W\left(0, \hat{T}_{n}\right) \subset \mathscr{D}$ where $W(0)$ is chosen uniformly on [0,2 $\pi i]$. In particular, if $Z_{n}^{l}$ and $O_{n}^{l}$ are defined as in Section 2.5, then $Z_{n}^{l}=n\left|\mu_{O_{n}^{l}}\right|$.

But conformal invariance shows that $\left|\mu_{\mathscr{D}}\right|=\left|\mu_{\mathscr{T}_{L}(\mathscr{D})}\right|$. On the other hand, we know that

$$
\mathbf{P}\left(W\left(0, \hat{T}_{m}\right) \subset \mathscr{T}_{m}\right) \approx e^{-m}, \quad m \rightarrow \infty
$$

when $W(0)$ is chosen uniformly on $[0,2 \pi i]$. 
As $L\left(O_{n}^{l}\right) \geq n / 2$, this implies that (uniformly with respect to the possible shapes of $O_{n}^{l}$ ),

$$
Z_{n}^{l}=n\left|\mu_{L\left(O_{n}^{l}\right)}\right| \approx \exp \left(-L\left(O_{n}^{l}\right)\right), \quad n \rightarrow \infty .
$$

Therefore, for any $\varepsilon>0$ and any large enough $n$,

$$
\begin{aligned}
s_{n}^{*}(\bar{p},(1-\varepsilon) \bar{\lambda}) & \geq \mathbf{E}^{*}\left[\exp \left\{-\sum_{l=1}^{k+1} \lambda_{l} L\left(O_{n}^{l}\right)\right\}\right] \\
& \geq s_{n}^{*}(\bar{p},(1+\varepsilon) \bar{\lambda}) .
\end{aligned}
$$

Combining this with the continuity of $\bar{\lambda} \mapsto \tilde{\xi}\left(\lambda_{1}, p_{1}, \ldots, p_{k}, \lambda_{k+1}\right)$ implies that

$$
\begin{aligned}
& \mathbf{E}^{*}\left(\exp \left\{-\sum_{l=1}^{k+1} \lambda_{l} L\left(O_{n}^{l}\right)\right\}\right) \\
& \quad \approx \exp \left(-n \tilde{\xi}\left(\lambda_{1}, p_{1}, \ldots, p_{k}, \lambda_{k+1}\right)\right), \quad n \rightarrow \infty .
\end{aligned}
$$

As we shall see, this is a very useful characterization of $\hat{\xi}$.

3.3. A first cascade relation. We are now going to use the results collected so far to show the following relation:

LEMMA 4. For all positive integers $p_{1}, \ldots, p_{k}$ and nonnegative reals $\lambda_{1}, \ldots, \lambda_{k+1}$,

$$
\tilde{\xi}\left(\lambda_{1}, p_{1}, \ldots, p_{k}, \lambda_{k+1}\right)=\tilde{\xi}\left(\lambda_{1}, p_{1}, \ldots, p_{k-1}, \tilde{\xi}\left(\lambda_{k}, p_{k}, \lambda_{k+1}\right)\right) .
$$

Proof. Throughout this proof, we fix $\bar{p}=\left(p_{1}, \ldots, p_{k}\right)$ and $\bar{\lambda}=$ $\left(\lambda_{1}, \ldots, \lambda_{k+1}\right)$ and assume $k \geq 2$. For convenience, we define

$$
\begin{aligned}
\bar{p}^{\prime} & =\left(p_{1}, \ldots, p_{k-1}\right), \\
K^{\prime} & =p_{1}+\cdots+p_{k-1}, \\
\tilde{\xi} & =\tilde{\xi}\left(\lambda_{1}, p_{1}, \ldots, p_{k}, \lambda_{k+1}\right), \\
\tilde{\xi}^{\prime \prime} & =\tilde{\xi}\left(\lambda_{k}, p_{k}, \lambda_{k+1}\right) .
\end{aligned}
$$

We define the domains $O_{n}^{1}, \ldots, O_{n}^{k+1}$ using the paths $W^{1}, \ldots, W^{K}$ as in previous sections. We also define $O_{n}^{*} \subset \mathscr{T}_{n}$ as being the path domain of length $n$ bounded below by $\Theta_{n}^{k-1}$ and above by $\left[i \pi, n+i \pi\right.$ ] (it is what $O_{n}^{k}$ would be if we were working with $\bar{p}^{\prime}$ instead of $\left.\bar{p}\right)$. Let $L_{n}^{*}=L\left(O_{n}^{*}\right)$.

In order to use conformal invariance efficiently, let us define the integrals $\mathbf{E}^{\prime}$ and $\mathscr{E}_{\mathscr{D}}^{\prime \prime}$ (when $\mathscr{D}$ is a path domain) as follows:

(i) $\mathbf{E}^{\prime}$ is the integral with respect to the probability measure for which $W^{1}, W^{2}, \ldots, W^{K^{\prime}}$ are independent CBMs started uniformly on $[0,2 \pi i]$.

(ii) $\mathscr{E}_{\mathscr{D}}^{\prime \prime}$ is the integral with respect to the measure for which $W^{K^{\prime}+1}, \ldots, W^{K}$ are independent processes each defined using the measure $\mu_{\mathscr{D}}$. 
The definition of $\mu_{\mathscr{T}_{n}}$ and (25) show immediately that

$$
\exp (-n \tilde{\xi}) \approx \mathbf{E}^{\prime}\left(\mathscr{E}_{\mathscr{T}_{n}}^{\prime \prime}\left(\exp \left\{-\sum_{l=1}^{k+1} \lambda_{l} L\left(O_{n}^{l}\right)\right\}\right)\right), \quad n \rightarrow \infty .
$$

Hence, when $n \rightarrow \infty$,

$$
\exp (-n \tilde{\xi}) \approx \mathbf{E}^{\prime}\left(\exp \left\{-\sum_{l=1}^{k-1} \lambda_{l} L\left(O_{n}^{l}\right)\right\} \mathscr{E}_{\mathscr{T}_{n}^{\prime \prime}}\left(\exp \left\{-\lambda_{k} L\left(O_{n}^{k}\right)-\lambda_{k+1} L\left(O_{n}^{k+1}\right)\right\}\right)\right) \text {. }
$$

But $L\left(O_{n}^{k}\right)$ and $L\left(O_{n}^{k+1}\right)$ are not infinite only if for all $j \in\left\{K^{\prime}+1, \ldots, K\right\}$,

$$
W^{j}\left(0, \hat{T}_{n}^{j}\right) \subset O_{n}^{*}
$$

so that we can replace $\mathscr{E}_{\mathscr{F}_{n}}^{\prime \prime}$ in the last expression by $\mathscr{E}_{O_{n}^{*}}^{\prime \prime}$. Conformal invariance implies that

$$
\begin{aligned}
& \mathscr{E}_{O_{n}^{*}}^{\prime \prime}\left(\exp \left\{-\lambda_{k} L\left(O_{n}^{k}\right)-\lambda_{k+1} L\left(O_{n}^{k+1}\right)\right\}\right) \\
& \quad=\mathscr{E}_{\mathscr{G}_{L^{*}}}^{\prime \prime}\left(\exp \left\{-\lambda_{k} L\left(O^{-}\right)-\lambda_{k+1} L\left(O^{+}\right)\right\}\right)
\end{aligned}
$$

where $\mathrm{O}^{-}$and $\mathrm{O}^{-}$are the two subpath domains of $\mathscr{T}_{L_{n}^{*}}$ of length $L_{n}^{*}$ that are, respectively, under and above all the paths $W^{K^{\prime}+1_{n}}, \ldots, W^{K}$. On the other hand

$$
\mathscr{E}_{\mathscr{G}_{L^{*}}}^{\prime \prime}\left(\exp \left\{-\lambda_{k} L\left(O^{-}\right)-\lambda_{k+1} L\left(O^{+}\right)\right\}\right) \approx \exp \left(-L_{n}^{*} \tilde{\xi}^{\prime \prime}\right), \quad L_{n}^{*} \rightarrow \infty .
$$

Finally, this shows that

$$
\begin{aligned}
\exp (-n \tilde{\xi}) & \approx \mathbf{E}^{\prime}\left(\exp \left\{-\sum_{l=1}^{k-1} \lambda_{l} L\left(O_{n}^{L}\right)\right\} \exp \left\{-L_{n}^{*} \tilde{\xi}^{\prime \prime}\right\}\right) \\
& \approx \exp \left(-n \tilde{\xi}\left(\lambda_{1}, p_{1}, \ldots, p_{k-1}, \tilde{\xi}^{\prime \prime}\right)\right), \quad n \rightarrow \infty .
\end{aligned}
$$

This implies Lemma 4.

3.4. Corresponding results for $\xi$. We have focused in the previous section on the exponents $\tilde{\xi}$. Similar arguments lead to the following first cascade relation for $\xi$. As this proof contains no new ideas, we simply leave it to the reader.

LEMma 5. For all positive integers $p_{1}, \ldots, p_{k}$ and nonnegative reals $\lambda_{1}, \ldots, \lambda_{k}$

$$
\xi\left(p_{1}, \lambda_{1}, p_{2}, \ldots, \lambda_{k}\right)=\xi\left(p_{1}, \lambda_{1}, \ldots, p_{k-1}, \tilde{\xi}\left(\lambda_{k-1}, p_{k}, \lambda_{k}\right)\right) .
$$

4. Relations between exponents. We will now put some results of Sections 2 and 3 together to obtain relations between exponents.

4.1. Cascade relations. We have already done most of the derivation of what we call the "cascade" relations. However, they are of sufficient importance that we restate them here. 
THEOREM 6. For any integers $1 \leq j<k$, for all positive integers $p_{1}, \ldots, p_{k}$ and nonnegative real numbers $\lambda_{1}, \ldots, \lambda_{k+1}$,

$$
\begin{aligned}
& \tilde{\xi}\left(\lambda_{1}, p_{1}, \lambda_{2}, \ldots, p_{k}, \lambda_{k+1}\right) \\
& \quad=\tilde{\xi}\left(\lambda_{1}, p_{1}, \ldots, p_{j}, \tilde{\xi}\left(\lambda_{j+1}, p_{j+1}, \ldots, p_{k}, \lambda_{k+1}\right)\right)
\end{aligned}
$$

and

$$
\xi\left(p_{1}, \lambda_{1}, p_{2}, \ldots, p_{k}, \lambda_{k}\right)=\xi\left(p_{1}, \lambda_{1}, \ldots, p_{j}, \tilde{\xi}\left(\lambda_{j}, p_{j+1}, \ldots, p_{k}, \lambda_{k}\right)\right) .
$$

Proof. Identity (26) in the case where $j=k-1$ has already been established; see Lemma 4.

One then gets that

$$
\begin{aligned}
\tilde{\xi}\left(\lambda_{1},\right. & \left.p_{1}, \ldots, p_{k}, \lambda_{k+1}\right) \\
& =\tilde{\xi}\left(\lambda_{1}, p_{1}, \ldots, p_{k-1}, \tilde{\xi}\left(\lambda_{k}, p_{k}, \lambda_{k+1}\right)\right) \\
& =\tilde{\xi}\left(\lambda_{1}, p_{1}, \ldots, p_{k-2}, \tilde{\xi}\left(\lambda_{k-1}, p_{k-1}, \tilde{\xi}\left(\lambda_{k}, p_{k}, \lambda_{k+1}\right)\right)\right) \\
& =\tilde{\xi}\left(\lambda_{1}, p_{1}, \ldots, p_{k-2}, \tilde{\xi}\left(\lambda_{k-1}, p_{k-1}, \lambda_{k}, p_{k}, \lambda_{k+1}\right)\right)
\end{aligned}
$$

and this proves (4) when $j=k-2$.

The general case is proved by induction.

Similarly, identity (27) follows from Lemma 5 and (26).

4.2. Commutation relations. The main result in this section is the following proposition.

Proposition 7. For all $k \geq 1$, for all positive integers $p_{1}, \ldots, p_{k}$ and for all nonnegative reals $\lambda_{1}, \ldots, \lambda_{k+1}$, for all permutations $\sigma$ and $\rho$ of $\{1, \ldots$, $k+1\}$ and $\{1, \ldots, k\}$, respectively,

$$
\tilde{\xi}\left(\lambda_{1}, p_{1}, \ldots, p_{k}, \lambda_{k+1}\right)=\tilde{\xi}\left(\lambda_{\sigma(1)}, p_{\rho(1)}, \lambda_{\sigma(2)}, \ldots, p_{\rho(k)}, \lambda_{\sigma(k+1)}\right) .
$$

Similarly, for all permutations $\tau$ and $\rho$ of $\{1, \ldots, k\}$,

$$
\xi\left(p_{1}, \lambda_{1}, \ldots, p_{k}, \lambda_{k}\right)=\xi\left(p_{\rho(1)}, \lambda_{\tau(1)}, \ldots, \lambda_{\tau(k-1)}, p_{\rho(k)}, \lambda_{\tau(k)}\right) .
$$

PRoOF. It is clear that for symmetry reasons

$$
\tilde{\xi}\left(\lambda_{1}, p_{1}, \ldots, p_{k}, \lambda_{k+1}\right)=\tilde{\xi}\left(\lambda_{k+1}, p_{k}, \lambda_{k}, \ldots, \lambda_{2}, p_{1}, \lambda_{1}\right) .
$$

Hence, for all $1 \leq l \leq k$, using the cascade relation,

$$
\begin{aligned}
\tilde{\xi}\left(\lambda_{1},\right. & \left.p_{1}, \ldots, p_{k}, \lambda_{k+1}\right) \\
& =\tilde{\xi}\left(\lambda_{1}, \ldots, p_{l-1}, \tilde{\xi}\left(\lambda_{l}, p_{l}, \ldots, \lambda_{k+1}\right)\right) \\
& =\tilde{\xi}\left(\lambda_{1}, \ldots, p_{l-1}, \tilde{\xi}\left(\lambda_{k+1}, p_{k}, \ldots, p_{l}, \lambda_{l}\right)\right) \\
& =\tilde{\xi}\left(\lambda_{1}, \ldots, p_{l-1}, \tilde{\xi}\left(\lambda_{k+1}, p_{k}, \ldots, p_{l+1}, \tilde{\xi}\left(\lambda_{l+1}, p_{l}, \lambda_{l}\right)\right)\right) \\
& =\tilde{\xi}\left(\lambda_{1}, \ldots, p_{l-1}, \tilde{\xi}\left(\lambda_{k+1}, p_{k}, \ldots, p_{l+1}, \tilde{\xi}\left(\lambda_{l}, p_{l}, \lambda_{l+1}\right)\right)\right)
\end{aligned}
$$




$$
\begin{aligned}
& =\cdots \\
& =\tilde{\xi}\left(\lambda_{1}, \ldots, p_{l-1}, \lambda_{l+1}, p_{l}, \lambda_{l}, p_{l+1}, \ldots, p_{k}, \lambda_{k+1}\right)
\end{aligned}
$$

so that (28) is true when $\rho=$ Identity and $\sigma$ is the transposition that swaps $l$ and $l+1$; this implies that (28) is true when $\rho=$ Identity and $\sigma$ is any permutation of $\{1, \ldots, k\}$.

In particular, for all $l \leq k-1$,

$$
\begin{aligned}
\tilde{\xi}\left(\lambda_{l}, p_{l}, \lambda_{l+1}, p_{l+1}, \lambda_{l+2}\right) & =\tilde{\xi}\left(\lambda_{l+2}, p_{l+1}, \lambda_{l+1}, p_{l}, \lambda_{l}\right) \\
& =\tilde{\xi}\left(\lambda_{l}, p_{l+1}, \lambda_{l+1}, p_{l}, \lambda_{l+2}\right)
\end{aligned}
$$

so that the same method as before easily shows that (28) is true when $\sigma=$ Identity and $\rho$ is the transposition that swaps $l$ and $l+1$. This implies that (28) holds for all permutations $\sigma$ and $\rho$.

To prove (29) we first recall that by symmetry, for all $2 \leq l \leq k$,

$$
\xi\left(p_{1}, \lambda_{1}, \ldots, \lambda_{k-1}, p_{k}, \lambda_{k}\right)=\xi\left(p_{l}, \lambda_{l}, \ldots, p_{k}, \lambda_{k}, p_{1}, \lambda_{1}, \ldots, p_{l-1}, \lambda_{l-1}\right) .
$$

Combining this with (28) and (27) easily implies (29).

4.3. Generalized exponents II. We are now going to give a precise meaning to

$$
\tilde{\xi}\left(\alpha_{1}, \ldots, \alpha_{k}\right)
$$

for all $\left(\alpha_{1}, \ldots, \alpha_{k}\right) \in \mathbb{R}_{+}^{k}$ and to

$$
\xi\left(\alpha_{1}, \ldots, \alpha_{k}\right)
$$

for all $\left(\alpha_{1}, \ldots, \alpha_{k}\right) \in \mathbb{R}_{+}$such that

$$
\alpha_{i} \geq 1, \alpha_{i^{\prime}} \geq 1 \text { for some } i \neq i^{\prime} \text {. }
$$

THEOREM 8. There exists a unique extension of the function $\tilde{\xi}\left(\alpha_{1}, \ldots, \alpha_{k}\right)$ for any $k \geq 1$ and $\alpha_{1}, \ldots, \alpha_{k} \geq 0$ such that:

(i) For any permutation $\sigma$ of $\{1, \ldots, k\}$,

$$
\tilde{\xi}\left(\alpha_{1}, \ldots, \alpha_{k}\right)=\tilde{\xi}\left(\alpha_{\sigma(1)}, \ldots, \alpha_{\sigma(k)}\right) .
$$

(ii) For any $2 \leq j \leq k$,

$$
\tilde{\xi}\left(\alpha_{1}, \ldots, \alpha_{k}\right)=\tilde{\xi}\left(\alpha_{1}, \ldots, \alpha_{j-1}, \tilde{\xi}\left(\alpha_{j}, \ldots, \alpha_{k}\right)\right) .
$$

Moreover, there exists also a unique extension of $\xi\left(\alpha_{1}, \ldots, \alpha_{k}\right)$ for any $k \geq 2$ and $\alpha_{1}, \ldots, \alpha_{k} \geq 0$ satisfying (30) such that:

(i) For any permutation $\sigma$ of $\{1, \ldots, k\}$,

$$
\xi\left(\alpha_{1}, \ldots, \alpha_{k}\right)=\xi\left(\alpha_{\sigma(1)}, \ldots, \alpha_{\sigma(k)}\right) .
$$

(ii) For any $2 \leq j \leq k$ such that $\alpha_{i} \geq 1$ for some $i<j$,

$$
\xi\left(\alpha_{1}, \ldots, \alpha_{k}\right)=\xi\left(\alpha_{1}, \ldots, \alpha_{j-1}, \tilde{\xi}\left(\alpha_{j}, \ldots, \alpha_{k}\right)\right) .
$$


Proof. Recall that $\tilde{\kappa}(\lambda)=\tilde{\xi}(1, \lambda)$ is bijective from $[0, \infty)$ onto $[1, \infty)$. For any nonnegative reals $\alpha$ and $\alpha^{\prime}, \tilde{\xi}\left(\alpha, 1, \alpha^{\prime}\right) \geq 1$, so that there exists a unique $\beta \geq 0$ such that

We now define

$$
\tilde{\xi}(1, \beta)=\tilde{\xi}\left(\alpha, 1, \alpha^{\prime}\right)
$$

$$
\tilde{\xi}\left(\alpha, \alpha^{\prime}\right):=\beta=\tilde{\kappa}^{-1}\left(\tilde{\xi}\left(\alpha, 1, \alpha^{\prime}\right)\right) .
$$

Note that this function $\tilde{\xi}\left(\alpha, \alpha^{\prime}\right)$ is continuous on $[0, \infty)^{2}$ [because both $\tilde{\kappa}^{-1}$ and $\left(\alpha, \alpha^{\prime}\right) \mapsto \tilde{\xi}\left(\alpha, 1, \alpha^{\prime}\right)$ are continuous].

In the case where one of the two values $\alpha$ or $\alpha^{\prime}$ is an integer, then the commutation and cascade relations imply that this new definition is consistent with our previous definitions.

Suppose now that $\alpha_{1}, \ldots, \alpha_{k}$ are nonnegative reals. We define inductively

$$
\tilde{\xi}\left(\alpha_{1}, \ldots, \alpha_{k}\right)=\tilde{\xi}\left(\alpha_{1}, \tilde{\xi}\left(\alpha_{2}, \ldots, \alpha_{k}\right)\right) .
$$

By induction, we see that $\tilde{\xi}$ is continuous on $[0, \infty)^{k}$ (for all $k \geq 2$ ). Again, the cascade and commutation relations ensure that this new definition is consistent. The (generalized) cascade relation

$$
\tilde{\xi}\left(\alpha_{1}, \ldots, \alpha_{k}\right)=\tilde{\xi}\left(\alpha_{1}, \ldots, \alpha_{j-1}, \tilde{\xi}\left(\alpha_{j}, \ldots, \alpha_{k}\right)\right)
$$

is immediate from the definition. The (generalized) commutation relation

$$
\tilde{\xi}\left(\alpha_{1}, \ldots, \alpha_{k}\right)=\tilde{\xi}\left(\alpha_{\sigma(1)}, \ldots, \alpha_{\sigma(k)}\right)
$$

(for any permutation $\sigma$ ) holds since repeated application of

gives

$$
\tilde{\xi}\left(1, \tilde{\xi}\left(\alpha_{1}, \ldots, \alpha_{k}\right)\right)=\tilde{\xi}\left(\alpha_{1}, 1, \tilde{\xi}\left(\alpha_{2}, \ldots, \alpha_{k}\right)\right)
$$

$$
\begin{aligned}
\tilde{\xi}\left(1,1, \ldots, 1, \tilde{\xi}\left(\alpha_{1}, \ldots, \alpha_{k}\right)\right) & =\tilde{\xi}\left(\alpha_{1}, 1, \alpha_{2}, \ldots, 1, \alpha_{k}\right) \\
& =\tilde{\xi}\left(\alpha_{\sigma(1)}, 1, \alpha_{\sigma(2)}, \ldots, 1, \alpha_{\sigma(k)}\right) \\
& =\tilde{\xi}\left(1, \ldots, 1, \tilde{\xi}\left(\alpha_{\sigma(1)}, \ldots, \alpha_{\sigma(k)}\right)\right) .
\end{aligned}
$$

We now define the exponents $\xi\left(\alpha_{1}, \ldots, \alpha_{k}\right)$. We will define $\xi$ only when two of the $\alpha_{i}$ 's is greater or equal to 1 . If $\alpha_{i} \geq 1$, we define $\beta_{i}$ by

We then define

$$
\alpha_{i}=\tilde{\xi}\left(1, \beta_{i}\right) \text {. }
$$

$$
\begin{aligned}
\xi\left(\alpha_{1}, \ldots, \alpha_{k}\right) & =\xi\left(1, \tilde{\xi}\left(\alpha_{1}, \ldots, \alpha_{i-1}, \beta_{i}, \alpha_{i+1}, \ldots, \alpha_{k}\right)\right) \\
& =\xi\left(1, \tilde{\xi}\left(\beta_{i}, \alpha_{1}, \ldots, \alpha_{i-1}, \alpha_{i+1}, \ldots, \alpha_{k}\right)\right)
\end{aligned}
$$

The second identity follows from the commutation relation for $\tilde{\xi}$. To see that this is well defined, note that if $\alpha_{i} \geq 1$ and $\alpha_{i^{\prime}} \geq 1$ for $i<i^{\prime}$, then the cascade and commutation relations for $\tilde{\xi}$ imply that $\xi\left(\alpha_{1}, \ldots, \alpha_{k}\right)$ equals

$$
\xi\left(1, \tilde{\xi}\left(1, \beta_{i}, \beta_{i^{\prime}}, \alpha_{1}, \ldots, \alpha_{i-1}, \alpha_{i+1}, \ldots, \alpha_{i^{\prime}-1}, \alpha_{i^{\prime}+1}, \ldots, \alpha_{k}\right)\right) .
$$


The generalized commutation relation for $\tilde{\xi}$ imply the generalized commutation relation for $\xi$,

$$
\xi\left(\alpha_{1}, \ldots, \alpha_{k}\right)=\xi\left(\alpha_{\sigma(1)}, \ldots, \alpha_{\sigma(k)}\right) .
$$

Also, we get the generalized cascade relation for $\xi$. For instance, if $\alpha_{1} \geq 1$, and $2 \leq j<k$,

$$
\begin{aligned}
\xi\left(\alpha_{1}, \ldots, \alpha_{k}\right) & =\xi\left(1, \tilde{\xi}\left(\beta_{1}, \alpha_{2}, \ldots, \alpha_{k}\right)\right) \\
& =\xi\left(1, \tilde{\xi}\left(\beta_{1}, \alpha_{2}, \ldots, \alpha_{j-1}, \tilde{\xi}\left(\alpha_{j}, \ldots, \alpha_{k}\right)\right)\right) \\
& =\xi\left(\alpha_{1}, \ldots, \alpha_{j-1}, \tilde{\xi}\left(\alpha_{j}, \ldots, \alpha_{k}\right)\right) .
\end{aligned}
$$

Finally, it is easy to check (using (27), that this definition of $\xi$ indeed generalizes our previous definitions of $\xi\left(p_{1}, \lambda_{1}, \ldots, p_{k}, \lambda_{k}\right)$. This is where the assumption (30) is needed: Suppose for instance that $p \geq 1$ and $\lambda \geq 1$ and let $\lambda=\tilde{\xi}(1, \beta)$, then using our initial definition of $\xi$ and the cascade relation (27), we indeed get that

$$
\xi(p, \lambda)=\xi(p, \tilde{\xi}(1, \beta))=\xi(p, \beta, 1)=\xi(1, \tilde{\xi}(p, \beta))
$$

Note that all the exponents $\tilde{\xi}$ can be derived from the values

$$
\tilde{\xi}(\alpha, 1, \beta), \quad \alpha \geq 0, \beta \geq 0,
$$

and all the exponents $\xi$ can be derived from $\tilde{\xi}$ and the values

We also define, as before,

$$
\xi(1, \beta), \quad \beta \geq 1 \text {. }
$$

$$
\hat{\xi}\left(\alpha_{1}, \ldots, \alpha_{k}\right)=\tilde{\xi}\left(\alpha_{1}, \ldots, \alpha_{k}\right)-\left(\alpha_{1}+\cdots+\alpha_{k}\right) .
$$

\subsection{Generalized disconnection exponents.}

THEOREM 9. There exists an increasing function $\eta:[\tilde{\xi}(1,1), \infty) \rightarrow \mathbb{R}_{+}$such that for any integer $k \geq 2$ and for all $\alpha_{1}, \ldots, \alpha_{k} \in \mathbb{R}_{+}^{k}$ such that (30),

$$
\xi\left(\alpha_{1}, \ldots, \alpha_{k}\right)=\eta\left(\tilde{\xi}\left(\alpha_{1}, \ldots, \alpha_{k}\right)\right) .
$$

PRoof. Recall that $\lambda \mapsto \xi(1, \lambda)$ is concave and increasing on $[0, \infty)$. In particular, it is continuous on $(0, \infty)$. Define for any $\lambda \geq 0$,

and recall that $\tilde{\kappa}(\lambda)=\tilde{\xi}(1, \lambda)$ is bijective.

$$
\kappa(\lambda)=\xi(1, \lambda)
$$

Assume for instance that $\alpha_{1} \geq 1$ and define $\beta_{1}=\tilde{\kappa}^{-1}\left(\alpha_{1}\right)$ so that $\tilde{\xi}\left(1, \beta_{1}\right)$ $=\alpha_{1}$.

$$
\begin{aligned}
\xi\left(\alpha_{1}, \ldots, \alpha_{k}\right) & =\xi\left(1, \tilde{\xi}\left(\beta_{1}, \alpha_{2}, \ldots, \alpha_{k}\right)\right) \\
& =\xi\left(1, \tilde{\kappa}^{-1}\left(\tilde{\xi}\left(1, \beta_{1}, \alpha_{2}, \ldots, \alpha_{k}\right)\right)\right) \\
& =\xi\left(1, \tilde{\kappa}^{-1}\left(\tilde{\xi}\left(\alpha_{1}, \ldots, \alpha_{k}\right)\right)\right) \\
& =\kappa \circ \tilde{\kappa}^{-1}\left(\tilde{\xi}\left(\alpha_{1}, \ldots, \alpha_{k}\right)\right) .
\end{aligned}
$$


Recall that $\tilde{\kappa}$ is continuous on $[1, \infty[$, and that we have seen (see the end of Section 2.5) that $\kappa$ is continuous on $(0, \infty)$. Hence, the function $\eta=\kappa \circ \tilde{\kappa}^{-1}$ is continuous on $[\tilde{\xi}(1,1), \infty)$.

\section{Asymptotic behavior.}

\subsection{A lower bound for $\tilde{\xi}$.}

LEMMA 10. For any nonnegative reals $\lambda_{1}, \ldots, \lambda_{k+1}$, $\tilde{\xi}\left(\lambda_{1}, 1, \lambda_{2}, 1, \lambda_{3}, \ldots, 1, \lambda_{k+1}\right) \geq \tilde{\xi}\left(1^{\otimes k}\right)+\left(\sqrt{\lambda_{1}}+\sqrt{\lambda_{2}}+\cdots+\sqrt{\lambda_{k+1}}\right)^{2}$.

Proof. Take $\bar{p}=1^{\otimes k}$, let $W^{1}, \ldots, W^{k}$ denote $k$ independent CBMs and define the subsets $O_{n}^{1}, O_{n}^{2}, \ldots, O_{n}^{k+1}$ the $k+1$ subsets of $\mathscr{T}_{n}$ between the CBMs as in the previous section. From our previous results, it suffices to get an upper bound for

$$
\mathbf{E}^{*}\left[1_{\hat{F}_{0} \cap \hat{E}_{n}} \exp \left\{-\lambda_{1} L\left(O^{1}\right)-\lambda_{2} L\left(O^{2}\right)-\cdots-\lambda_{k+1} L\left(O^{k+1}\right)\right\}\right]
$$

in order to find a lower bound for $\tilde{\xi}\left(\lambda_{1}, 1, \ldots, 1, \lambda_{k+1}\right)$. But Lemma 3 shows that

$$
\sum_{l=1}^{k+1} \lambda_{l} L\left(O^{l}\right) \geq n\left(\sqrt{\lambda_{1}}+\cdots+\sqrt{\lambda_{k+1}}\right)^{2}
$$

and this readily implies the lemma.

5.2. Asymptotic behavior: first approach. In the present section, we are going to study the asymptotic behavior of the intersection exponents when the number of packets tends to infinity. When $N>0$ and $\alpha \geq 0$, we define

$$
\alpha^{\otimes N}=(\alpha, \ldots, \alpha) \in \mathbb{R}_{+}^{N} .
$$

Also, if $\bar{a} \in \mathbb{R}_{+}^{k}$, we define

$$
\bar{a}^{\otimes N}=(\bar{a}, \ldots, \bar{a}) \in \mathbb{R}_{+}^{k N} .
$$

The cascade relations for $\tilde{\xi}$ imply that

$$
\tilde{\xi}\left(\bar{a}^{\otimes N}\right)=\tilde{\xi}\left(\tilde{\xi}(\bar{a})^{\otimes N}\right) .
$$

We are going to study the asymptotic behavior of $\tilde{\xi}\left(\bar{a}^{\otimes N}\right)$ when $N \rightarrow \infty$. This type of approach is reminiscent of what physicists call "replica method." We shall prove the following result:

THEOREM 11. There exists a function $U: \mathbb{R}_{+} \rightarrow \mathbb{R}_{+}$such that for all $\bar{a} \in \mathbb{R}_{+}^{k}$,

$$
\lim _{N \rightarrow \infty} \frac{1}{N^{2}} \tilde{\xi}\left(\bar{a}^{\otimes N}\right)=U^{2}(\tilde{\xi}(\bar{a})) .
$$

Note that this result is of a different type than that derived in [35], which we will recall and use in the next subsection, where the number of packets is constant and the number of Brownian motions per packet tends to infinity. 
Proof. Because of (32), it suffices to consider the case where $\bar{a}=\alpha \in$ $[0, \infty)$.

We are first going to prove suppose that $\alpha \geq 1$. Define $\beta=\tilde{\kappa}^{-1}(\alpha)$ so that $\alpha=\tilde{\xi}(1, \beta)$. Let

$$
\zeta_{N}=\zeta_{N}(\beta):=\tilde{\xi}\left((1, \beta)^{\otimes N}\right)
$$

Then, using Lemma 10, we get that for any positive integers $k, l$,

$$
\zeta_{k+l+1} \geq \tilde{\xi}\left(\zeta_{l}, 1, \beta, \zeta_{k}\right) \geq \tilde{\xi}\left(\zeta_{l}, 1, \zeta_{k}\right) \geq\left(\sqrt{\zeta_{l}}+\sqrt{\zeta_{k}}\right)^{2} .
$$

Now, define for all $k>1, b_{k}=\sqrt{\zeta_{k-1}}$. Then, for all $k>1$ and $l>1$,

$$
b_{k+l}=\sqrt{\zeta_{(k-1)+(l-1)+1}} \geq \sqrt{\zeta_{k-1}}+\sqrt{\zeta_{l-1}}=b_{k}+b_{l} .
$$

Define

$$
U(\alpha):=\sup _{n>1} \frac{b_{n}}{n} .
$$

This quantity is finite as

$$
\zeta_{n} \leq n^{2}(1+\sqrt{\beta})^{2}
$$

[see (19)]. Finally,

$$
\lim _{n \rightarrow \infty} \frac{\zeta_{n}}{n^{2}}=\lim _{n \rightarrow \infty} \frac{b_{n}^{2}}{n^{2}}=U^{2}(\alpha) .
$$

We now suppose that $\alpha \in(0,1)$ [the case $U(0)=0$ is trivial]. It follows from (20) that

so that

$$
\begin{aligned}
\tilde{\xi}\left(\alpha^{\otimes k}, 1\right) & \geq \tilde{\xi}\left(\tilde{\xi}\left(\alpha^{\otimes k-1}\right), 1, \alpha\right) \\
& \geq \tilde{\xi}\left(\alpha^{\otimes k-1}, 1\right)+\alpha \\
& \geq \cdots \\
& \geq 1+k \alpha
\end{aligned}
$$

$$
\lim _{k \rightarrow \infty} \tilde{\xi}\left(1, \tilde{\xi}\left(\alpha^{\otimes k}\right)\right)=\infty
$$

and $\left(\operatorname{as~} \lim _{+\infty} \tilde{\kappa}=+\infty\right)$, there exists $k_{0}=k_{0}(\alpha)$ such that

$$
\tilde{\xi}\left(\alpha^{\otimes k_{0}}\right) \geq 1 .
$$

However,

$$
\begin{aligned}
\lim _{N \rightarrow \infty} \frac{1}{\left(k_{0} N\right)^{2}} \tilde{\xi}\left(\alpha^{\otimes k_{0} N}\right) & =\frac{1}{k_{0}^{2}} \lim _{N \rightarrow \infty} \frac{1}{N^{2}} \tilde{\xi}\left(\left[\alpha^{\otimes k_{0}}\right]^{\otimes N}\right) \\
& =\frac{1}{k_{0}^{2}} U^{2}\left(\tilde{\xi}\left(\alpha^{\otimes k_{0}}\right)\right) .
\end{aligned}
$$


We then define

so that

$$
U(\alpha)=\frac{1}{k_{0}} U\left(\tilde{\xi}\left(\alpha^{\otimes k_{0}}\right)\right)
$$

$$
\lim _{N \rightarrow \infty} \frac{1}{N^{2}} \tilde{\xi}\left(\alpha^{\otimes N}\right)=U^{2}(\alpha) .
$$

We will derive some properties of the function $U$ in Section 5.4.

5.3. A functional relation. We are now going to derive the following result.

THEOREM 12. For all $\left(\alpha_{1}, \ldots, \alpha_{k}\right) \in \mathbb{R}_{+}^{k}$,

$$
U\left(\tilde{\xi}\left(\alpha_{1}, \ldots, \alpha_{k}\right)\right)=U\left(\alpha_{1}\right)+\cdots+U\left(\alpha_{k}\right) .
$$

This will be an easy consequence of the results that we have derived so far and of another asymptotic result that we now state and prove.

Proposition 13. For any positive reals $u_{1}, \ldots, u_{k}$,

$$
\lim _{M \rightarrow \infty} \frac{\tilde{\xi}\left(M u_{1}, \ldots, M u_{k}\right)}{M}=\left(\sqrt{u_{1}}+\cdots+\sqrt{u_{k}}\right)^{2} .
$$

This result was already derived in [35].

Proof. Fix $k \geq 2$ and $u_{1}, \ldots, u_{k}>0$. Lemma 10 and (19) show that

so that

$$
\begin{aligned}
\left(k+\sqrt{M u_{1}}+\cdots+\sqrt{M u_{k}}\right)^{2} & \geq \tilde{\xi}\left(M u_{1}, 1, M u_{2}, 1, \ldots, M u_{k}, 1,0\right) \\
& \geq\left(\sqrt{M u_{1}}+\cdots+\sqrt{M u_{k}}\right)^{2}
\end{aligned}
$$

$$
\tilde{\xi}\left(M u_{1}, 1, M u_{2}, 1, \ldots, M u_{k}, 1\right) \sim M\left(\sqrt{u_{1}}+\sqrt{u_{2}}+\cdots+\sqrt{u_{k}}\right)^{2}
$$

when $M \rightarrow \infty$. But (21) and the cascade relations imply that

$$
\tilde{\xi}\left(1^{\otimes k}, \lambda\right) \sim \tilde{\xi}\left(1^{\otimes k-1}, \lambda\right) \sim \cdots \sim \lambda
$$

when $\lambda \rightarrow \infty$. Hence,

$$
\tilde{\xi}\left(M u_{1}, \ldots, M u_{k}\right) \sim \tilde{\xi}\left(1^{\otimes k}, \tilde{\xi}\left(M u_{1}, \ldots, M u_{k}\right)\right) \sim M\left(\sqrt{u_{1}}+\cdots+\sqrt{u_{k}}\right)^{2}
$$

when $M \rightarrow \infty$.

Proof of Theorem 12. Let us fix $\alpha_{1}, \ldots, \alpha_{k}$ and define $\gamma=\tilde{\xi}\left(\alpha_{1}, \ldots, \alpha_{k}\right)$. We have seen that

$$
\lim _{N \rightarrow \infty} \frac{\tilde{\xi}\left(\gamma^{\otimes N}\right)}{N^{2}}=U^{2}(\gamma)
$$


But the commutation and cascade relations show that

$$
\tilde{\xi}\left(\gamma^{\otimes N}\right)=\tilde{\xi}\left(\tilde{\xi}\left(\alpha_{1}^{\otimes N}\right), \ldots, \tilde{\xi}\left(\alpha_{k}^{\otimes N}\right)\right),
$$

and (33) then implies that for all $\varepsilon>0$, there exists $N_{0}$ such that for all $N>N_{0}$ and all $j \in\{1, \ldots, k\}$,

$$
N^{2}\left(U\left(\alpha_{j}\right)-\varepsilon\right)^{2} \leq \tilde{\xi}\left(\alpha_{j}^{\otimes N}\right) \leq N^{2}\left(U\left(\alpha_{j}\right)+\varepsilon\right)^{2} .
$$

Hence, combining this with (35) shows that

$$
\liminf _{N \rightarrow \infty} \frac{\tilde{\xi}\left(\gamma^{\otimes N}\right)}{N^{2}} \geq\left(U\left(\alpha_{1}\right)-\varepsilon+U\left(\alpha_{2}\right)-\varepsilon+\cdots+U\left(\alpha_{k}\right)-\varepsilon\right)^{2}
$$

and

$$
\limsup _{N \rightarrow \infty} \frac{\tilde{\xi}\left(\gamma^{\otimes N}\right)}{N^{2}} \leq\left(U\left(\alpha_{1}\right)+\varepsilon+U\left(\alpha_{2}\right)+\varepsilon+\cdots+U\left(\alpha_{k}\right)+\varepsilon\right)^{2}
$$

so that finally,

$$
U^{2}(\gamma)=\lim _{N \rightarrow \infty} \frac{\tilde{\xi}\left(\gamma^{\otimes N}\right)}{N^{2}}=\left(U\left(\alpha_{1}\right)+\cdots+U\left(\alpha_{k}\right)\right)^{2}
$$

and this proves the theorem.

5.4. Simple properties of $U$. Let us now make a list of some simple properties of the function $U$.

Proposition 14.

(i) $U$ is continuous and strictly increasing on $[0, \infty)$.

(ii) The function $\alpha \mapsto(U(1)+U(\alpha))^{2}$ is concave.

(iii) The function $U$ is strictly concave.

(iv) When $x \rightarrow \infty, U(x) \sim \sqrt{x}$.

PRoof. Theorem 12 immediately implies that $U$ is strictly increasing as $U(\alpha)>0$ for all $\alpha>0$ and $\tilde{\xi}$ is continuous and strictly increasing in all variables.

Let us now show that $U$ is continuous. First, note that for any $\alpha \geq 0$,

$$
(U(1)+U(\alpha))^{2}=U^{2}(\tilde{\xi}(1, \alpha))=\lim _{N \rightarrow \infty} \frac{\tilde{\xi}\left((1, \alpha)^{\otimes N}\right)}{N^{2}} .
$$

We know that for any fixed $N, \alpha \mapsto \tilde{\xi}\left((1, \alpha)^{\otimes N}\right)$ is a concave function, so that $(U(1)+U(\alpha))^{2}$ is also a concave function of $\alpha$ (as a limit of concave functions). Hence, $\alpha \mapsto U(\alpha)$ is continuous on $(0, \infty)$.

On the other hand,

$$
U(\alpha)=U(\tilde{\xi}(1, \alpha))-U(1)
$$

so that ( $U$ is continuous at 1 , and $\tilde{\kappa}$ is continuous at 0$) U$ is also continuous at 0 . 
Note the fact that $(U(1)+U(\alpha))^{2}$ is a concave function of $\alpha$ implies that $U$ is also a concave function. As $U$ is strictly increasing, it actually implies that $U$ is strictly concave.

Let us now prove that $U(x) \sim \sqrt{x}$. Recall that

This implies that

$$
\tilde{\xi}\left((1, \beta)^{\otimes N}\right) \leq N^{2}(1+\sqrt{\beta})^{2} .
$$

$$
U^{2}(\tilde{\xi}(1, \beta)) \leq(1+\sqrt{\beta})^{2} .
$$

Since $\tilde{\xi}(1, \beta) \sim \beta$ [see $(21)]$,

$$
\limsup _{\beta \rightarrow \infty} \frac{U^{2}(\beta)}{\beta} \leq 1 .
$$

On the other hand, fix a (large) integer $k$, and define $\varepsilon=1 / k, x_{0}=\tilde{\xi}\left(1^{\otimes k}\right)$. Then, for any $x \geq x_{0}$, and for any $N=k N^{\prime}>0$,

$$
\begin{aligned}
\tilde{\xi}\left(x^{\otimes N(1+\varepsilon)}\right) & \geq \tilde{\xi}\left(x^{\otimes N}, x_{0}^{\otimes N^{\prime}}\right) \\
& =\tilde{\xi}\left(x^{\otimes N}, 1^{\otimes N}\right) \\
& \geq \tilde{\xi}\left(1^{\otimes N}\right)+N^{2} x \\
& \geq N^{2} x
\end{aligned}
$$

(we used Lemma 10 in the third line) so that for all $x \geq x_{0}, U^{2}(x) \geq$ $x /(1+\varepsilon)^{2}$. Combining this with (36) concludes the proof of the proposition.

As $U$ is a bijection from $\mathbb{R}_{+}$onto $\mathbb{R}_{+}$, we can define $U^{-1}$ on $\mathbb{R}^{+}$and we can rewrite (34) and (31) as

and

$$
\tilde{\xi}\left(\alpha_{1}, \ldots, \alpha_{k}\right)=U^{-1}\left(U\left(\alpha_{1}\right)+\cdots+U\left(\alpha_{k}\right)\right)
$$

$$
\xi\left(\alpha_{1}, \ldots, \alpha_{k}\right)=\eta \circ U^{-1}\left(U\left(\alpha_{1}\right)+\cdots+U\left(\alpha_{k}\right)\right) .
$$

\section{Asymptotic behavior via the rate function.}

6.1. Rate functions. In this section we discuss some general basic facts about rate functions and Legendre transforms that we will use in Section 6.3. Suppose $U_{n}, V_{n}$ are sequences of random variables taking values in $[0,1]$. Suppose that there exists a function $\Xi:(0, \infty) \rightarrow(0, \infty)$ such that

$$
\mathbf{E}\left[V_{n}^{\lambda} U_{n}\right] \approx \exp (-n \Xi(\lambda)), \quad n \rightarrow \infty .
$$

Define the rate function $b:(0, \infty) \rightarrow[0, \infty]$ by

$$
b(a)=\liminf _{n \rightarrow \infty} \frac{-\log \mathbf{E}\left[U_{n} ; V_{n} \geq e^{-a n}\right]}{n} .
$$

It is standard and easy to show that if (37) holds, then

$$
\Xi(\lambda)=\inf _{a>0}\{a \lambda+b(a)\} \text {. }
$$


LEMMA 15. Let $U_{n}, V_{n}, \Xi, b$ be as above and assume that (37) holds. Suppose for some $a_{0} \geq 0$ and some $C>0$,

$$
\lim _{a \downarrow a_{0}}\left(a-a_{0}\right) b(a)=C .
$$

Then as $\lambda \rightarrow \infty$,

$$
\Xi(\lambda)=a_{0} \lambda+2 \sqrt{C} \sqrt{\lambda}+o(\sqrt{\lambda}) .
$$

Proof. Since $b(a) \rightarrow \infty$ as $a \downarrow a_{0}$, it follows from (38) that for all $\varepsilon>0$, if $\lambda$ is sufficiently large,

$$
\inf _{a>a_{0}}\left\{\left(a-a_{0}\right) \lambda+\frac{C-\varepsilon}{a-a_{0}}\right\} \leq \Xi(\lambda)-a_{0} \lambda \leq \inf _{a>a_{0}}\left\{\left(a-a_{0}\right) \lambda+\frac{C+\varepsilon}{a-a_{0}}\right\} .
$$

By taking the infimums of the two sides of this inequality, we get

$$
2 \sqrt{C-\varepsilon} \sqrt{\lambda} \leq \Xi(\lambda)-a_{0} \lambda \leq 2 \sqrt{C+\varepsilon} \sqrt{\lambda} .
$$

LemMa 16. Suppose the conditions of Lemma 15 hold with $a_{0}=1$. Suppose $\zeta_{n}$ is a sequence of real numbers going to infinity with

Then, as $n \rightarrow \infty$,

$$
\zeta_{n+1}=\Xi\left(\zeta_{n}\right) \text {. }
$$

$$
\zeta_{n} \sim C n^{2} \text { and } \zeta_{n+1}-\zeta_{n} \sim 2 C n
$$

Proof. Lemma 15 implies that

$$
\zeta_{n+1}-\zeta_{n}=2 \sqrt{C} \sqrt{\zeta_{n}}+o\left(\sqrt{\zeta_{n}}\right)
$$

The lemma follows easily from this difference inequality.

The Legendre transform is a way to "invert" the relation (38) to obtain a formula for $b$ in terms of $\Xi$. This cannot always be done. However, assume that $\Xi(\lambda)$ exists and is a $C^{2}$ function of $\lambda$ with

$$
\Xi^{\prime \prime}(\lambda)<0, \quad \lambda>0 .
$$

In particular $\Xi^{\prime}(\lambda)$ is a continuous, strictly decreasing function whose range is $\left(\Xi^{\prime}(\infty), \Xi^{\prime}(0)\right)$. In this case, it is not difficult to see that for $a \in\left(\Xi^{\prime}(\infty), \Xi^{\prime}(0)\right)$,

$$
b(a)=\sup _{\lambda}\{\Xi(\lambda)-a \lambda\} .
$$

In fact, the supremum is taken on at the $\lambda_{a}$ that satisfies $\Xi^{\prime}\left(\lambda_{a}\right)=a$.

6.2. Rate function for $\xi(p, \cdot)$. We now recall some results derived in [22]. Let $p$ be a positive integer, and let

$$
\xi_{p}(\lambda)=\xi(p, \lambda)
$$

and consider this as a function of $\lambda$. In [22], it was shown that $\xi_{p}(\lambda)$ is a $C^{2}$ function of $\lambda$ for $\lambda>0$ with

$$
\xi_{p}^{\prime \prime}(\lambda)<0, \quad \lambda>0
$$


Also,

$$
\lim _{\lambda \downarrow 0} \xi_{p}(\lambda)=\Xi_{p}(0)
$$

With the strict concavity of $\xi_{p}$, we can define two other important functions. Let

$$
a_{p}(\lambda)=\xi_{p}^{\prime}(\lambda)
$$

Note that $a_{p}(\lambda)$ is a continuous, strictly decreasing function. Also, if $Z_{n}=Z_{n}^{1}$ is as in Section 2.5, we define $b_{p}(a)$ by the relations

$$
\mathbf{P}^{*}\left\{Z_{n} \geq e^{-a n}\right\} \approx \exp \left(-n b_{p}(a)\right) .
$$

Then,

$$
\xi_{p}(\lambda)=\inf _{a}\left\{\lambda a+b_{p}(a)\right\}=\lambda a_{p}(\lambda)+b_{p}\left(a_{p}(\lambda)\right)
$$

6.3. Rate function for $\tilde{\xi}$. For this section, we fix $\beta \geq 1$, we choose $\lambda_{1} \geq 0$ such that $\tilde{\xi}\left(\lambda_{1}, 1\right)=\beta$ and we consider the function

$$
\Xi(\lambda):=\tilde{\xi}(\beta, \lambda)=\tilde{\xi}\left(\lambda_{1}, 1, \lambda\right) .
$$

We expect that the methods of [22] can be used to show that this is a strictly concave function of $\lambda$; however, since we will not need this result we will not bother to prove it. Instead we use the weaker "lim inf" definition of the rate function which will suffice for our purposes.

We will use the notation of previous sections which we review here. We let $W$ be a conditioned Brownian motion starting at a point chosen uniformly on the interval $[0,2 i \pi]$ and let

$$
\Theta_{n}=\Theta_{n}^{1}=W^{1}\left[0, \hat{T}_{n}^{1}\right] .
$$

Let $\hat{J}_{n}$ be the event

$$
\hat{J}_{n}=\left\{\Theta_{n} \subset \mathscr{T}_{n}\right\}
$$

On the event $\hat{J}_{n}$ we let $O_{n}^{1}, O_{n}^{2}$ be the "lower" and "upper" connected components, respectively, on $\mathscr{T}_{n} \backslash \Theta_{n}$ and we put $L_{n}=L\left(O_{n}^{2}\right)$ and $L_{n}^{1}=L\left(O_{n}^{1}\right)$. Then, as we have seen,

$$
\mathbf{E}^{*}\left[\left(\exp -\lambda_{1} L_{n}^{1}\right) \exp \left(-\lambda L_{n}\right)\right] \approx \exp (-\Xi(\lambda) n), \quad n \rightarrow \infty .
$$

To match the notation of Section 6.1 , let

$$
U_{n}=\exp \left(-\lambda_{1} L_{n}^{1}\right), \quad V_{n}=\exp \left(-L_{n}\right)
$$

so that

$$
\mathbf{E}^{*}\left[V_{n}^{\lambda} U_{n}\right] \approx \exp (-\Xi(\lambda) n), \quad n \rightarrow \infty .
$$

Here the expectation is with respect to the measure on $W$ assuming that the initial point is chosen uniformly on $[0,2 \pi i]$. 
As in Section 6.1, we define the rate function $\tilde{b}_{\beta}(a)$ by

$$
\tilde{b}_{\beta}(a)=\liminf _{n \rightarrow \infty} \frac{-\log \mathbf{E}^{*}\left[U_{n} ; V_{n} \geq e^{-n a}\right]}{n}=\liminf _{n \rightarrow \infty} \frac{-\log \mathbf{E}^{*}\left[U_{n} ; L_{n} \leq n a\right]}{n} \text {. }
$$

We expect that we could prove this limit exists, but since we do not need this fact, we will not go through the effort. By (38),

$$
\tilde{\xi}(\beta, \lambda)=\Xi(\lambda)=\inf _{a}\left\{a \lambda+\tilde{b}_{\beta}(a)\right\} .
$$

It is easy to check that $\tilde{b}_{\beta}(a)<\infty$ for $a>1$ and

Define the function

$$
\lim _{a \downarrow 1} \tilde{b}_{\beta}(a)=\infty .
$$

for all $a>0$.

$$
g_{\beta}(a)=\frac{a}{a+1} \tilde{b}_{\beta}(a+1)
$$

Proposition 17. For every $\beta \geq 1, g_{\beta}$ is an increasing function for $a>0$. Moreover,

$$
\lim _{a \downarrow 0} g_{\beta}(a)>0 .
$$

Proof. Fix $\beta \geq 1$ and define $\tilde{b}=\tilde{b}_{\beta}$ and $g=g_{\beta}$. Estimates for rectangles can be used to show that

$$
\liminf _{a \downarrow 0} g(a)>0,
$$

so the second conclusion follows once we show that $g$ is an increasing function.

For $0<\alpha \leq 1$, let $\mathscr{T}_{n, \alpha}$ denote the $n \times \alpha \pi$ rectangle

$$
\mathscr{T}_{n, \alpha}=\{x+i y: 0<x<n \text { and } 0<y<\alpha \pi\} \text {. }
$$

Let $J_{n, \alpha}$ be the event

$$
J_{n, \alpha}=\left\{\Theta_{n} \subset \mathscr{T}_{n, \alpha}\right\},
$$

so that $J_{n}=J_{n, 1}$. On the event $J_{n, \alpha}$, let $L_{n, \alpha}^{*}$ be $\pi$ times the extremal distance of the subdomain $\mathscr{T}_{n, \alpha}^{*}$ of $\mathscr{T}_{n, \alpha}$ bounded below by the Brownian path, above by a subinterval of $\{\mathfrak{F}(z)=\alpha \pi\}$, on the left by a subinterval of $\{\Re(z)=0\}$ and on the right by a subinterval of $\{\Re(z)=n\}$. By standard composition laws for extremal distances (see [1], Theorem 4.2), if the event $J_{n \alpha}$ is satisfied, then,

$$
L_{n} \leq\left[\left(L_{n, \alpha}^{*}\right)^{-1}+\left(\frac{1-\alpha}{n}\right)\right]^{-1}
$$

(i.e., a consequence of the fact that the rectangle $[0, n] \times[\alpha \pi, \pi]$ is "above" the set $\mathscr{T}_{n, \alpha}^{*}$ and that $O_{n}^{2}$ is the union of these two sets). But by conformal invariance, if we multiply by $\alpha^{-1}$,

$$
\mathbf{E}^{*}\left[U_{\alpha n} ; L_{\alpha n, \alpha}^{*} \leq(1+a) n ; J_{\alpha n, \alpha}\right]=\alpha \mathbf{E}^{*}\left[U_{n} ; L_{n} \leq(1+a) n ; J_{n}\right] .
$$


From above, if $L_{\alpha n, \alpha}^{*} \leq(1+a) n$, then

$$
L_{\alpha n} \leq \alpha n\left[1+\frac{\alpha a}{1+a(1-\alpha)}\right] \text {. }
$$

Hence,

$$
\mathbf{E}^{*}\left[U_{\alpha n} ; L_{\alpha n} \leq \alpha n\left(1+\frac{\alpha a}{1+a(1-\alpha)}\right)\right] \geq \alpha \mathbf{E}^{*}\left[U_{n} ; L_{n} \leq(1+a) n ; J_{n}\right] .
$$

Taking the appropriate lim inf on both sides we get

$$
\tilde{b}\left(1+\frac{\alpha a}{1+a(1-\alpha)}\right) \leq \alpha^{-1} \tilde{b}(1+a) .
$$

Since any $a^{\prime} \in(0, a)$ can be written as

$$
a^{\prime}=\frac{\alpha a}{1+a(1-\alpha)},
$$

for some $\alpha \in(0,1)$, we see that

$$
g\left(a^{\prime}\right)=\frac{a^{\prime}}{1+a^{\prime}} \tilde{b}\left(1+a^{\prime}\right) \leq \frac{\alpha a}{1+a} \tilde{b}(1+a)=g(a)
$$

and the proposition is proved.

As a corollary to Proposition 17, we give another proof of the existence of the limit (33) and of the slightly stronger statement

$$
\lim _{n \rightarrow \infty} \frac{\tilde{\xi}\left(\beta^{\otimes n+1}\right)-\tilde{\xi}\left(\beta^{\otimes n}\right)}{n}=2 U^{2}(\beta) .
$$

Let $\zeta_{n}=\tilde{\xi}\left(\beta^{\otimes n}\right)=\tilde{\xi}\left(\left(1, \lambda_{1}\right)^{\otimes n}\right)$. Clearly $\zeta_{n} \rightarrow \infty$, and the cascade relation implies

$$
\zeta_{n+1}=\Xi\left(\zeta_{n}\right)
$$

But Lemmas 15 and 16 then imply

where

$$
\lim _{n \rightarrow \infty} \frac{\zeta_{n}}{n^{2}}=C \quad \text { and } \lim _{n \rightarrow \infty} \frac{\zeta_{n+1}-\zeta_{n}}{n}=2 C,
$$

$$
C=C_{\beta}=\lim _{a \downarrow 0} g_{\beta}(a)=\lim _{a \downarrow 1} \frac{a-1}{a} \tilde{b}_{\beta}(a) .
$$

Recall that we denote this limit as $U^{2}(\beta)$. Note that as $g$ is increasing,

$$
\tilde{b}_{\beta}(a) \geq \frac{a}{a-1} U^{2}(\beta) .
$$

REMARK. Using the same ideas, it is easy to define $b_{\beta}$ for all $\beta>1$ that corresponds to the rate function for $\xi(\beta, \cdot)$. This generalizes the function $b_{p}$ described in Section 6.2. 
7. Conjectures. In this section, we will show that Duplantier-Kwon's conjectures [10] on the values of $\xi\left(1^{\otimes N}\right)$ and $\tilde{\xi}\left(1^{\otimes N}\right)$ in fact lead to more general conjectures for all exponents.

7.1. Intersection exponents in half-space. Duplantier-Kwon (see [10, 11]) studied the intersection exponents in the half-space and made the conjecture

$$
\hat{\xi}\left(1^{\otimes N}\right)=\frac{2\left(N^{2}-N\right)}{3},
$$

which is equivalent to the conjecture

$$
\tilde{\xi}\left(1^{\otimes N}\right)=\frac{N(2 N+1)}{3} .
$$

Hence, if we put

$$
f(x)=\frac{x(2 x+1)}{3}=\frac{(4 x+1)^{2}-1}{24}
$$

then, for all $n \geq 0, p \geq 0$,

$$
\tilde{\xi}(f(n), f(p))=\tilde{\xi}\left(1^{\otimes n}, 1^{\otimes p}\right)=\tilde{\xi}\left(1^{\otimes n+p}\right)=f(n+p) .
$$

If we now assume that this equation also holds for noninteger $p$ 's, comparing this with the functional relation (34) shows that for some constant $c>0$, $U(y)=c f^{-1}(y)$. But Duplantier's conjecture (42) shows that $U^{2}(1)=2 / 3$, so that

$$
U(x)=\sqrt{x+(1 / 24)}-\sqrt{1 / 24}=\frac{\sqrt{24 x+1}-1}{\sqrt{24}}
$$

and

$$
U^{-1}(y)=(y+\sqrt{1 / 24})^{2}-\frac{1}{24}=\frac{(\sqrt{24} y+1)^{2}-1}{24}
$$

and therefore, for all $\alpha_{1}, \ldots, \alpha_{k}$,

$$
\tilde{\xi}\left(\alpha_{1}, \ldots, \alpha_{k}\right)=\frac{\left(\sqrt{24 \alpha_{1}+1}+\sqrt{24 \alpha_{2}+1}+\cdots+\sqrt{24 \alpha_{k}+1}-(k-1)\right)^{2}-1}{24} .
$$

When $\alpha_{1}=\cdots=\alpha_{k}=1$, we of course recover (42). Note that some exponents are not rational numbers (see the remark in Section 7.3).

Let us choose $\beta \geq 1$ and use the notation of Section 6.3. Then a particular case of the conjecture is

$$
\tilde{\xi}(\beta, \lambda)=\left[U(\beta)+U(\lambda)+\frac{1}{\sqrt{24}}\right]^{2}-\frac{1}{24} .
$$

Note that for fixed $\beta$, this is a strictly concave function of $\lambda$. Hence, by (39), the conjecture implies a conjecture for the rate function,

$$
\tilde{b}_{\beta}(a)=\sup _{\lambda}\{\tilde{\xi}(\beta, \lambda)-a \lambda\}=U^{2}(\beta) \frac{a}{a-1}+\frac{a-1}{24} .
$$

One might wish to compare this with the rigorous estimate (41). 
Note that the conjecture gives the following intriguing conjecture for the conditioned exponent:

$$
\hat{\xi}\left(\alpha_{1}, \alpha_{2}\right)=2 U\left(\alpha_{1}\right) U\left(\alpha_{2}\right) .
$$

7.2. Intersection exponents. We have seen that for all $\alpha_{1}, \ldots, \alpha_{k}$, such that [30]

$$
\xi\left(\alpha_{1}, \ldots, \alpha_{k}\right)=V\left(U\left(\alpha_{1}\right)+\cdots+U\left(\alpha_{k}\right)\right)
$$

for some increasing function $V=\eta \circ U^{-1}$. Again, the conjectures for $\xi\left(1^{\otimes N}\right)$ will be sufficient to derive the general conjecture for $V$. Indeed, from the conjecture

$$
\xi\left(1^{\otimes N}\right)=\frac{4 N^{2}-1}{12}
$$

we get that for all $N \geq 1$,

$$
V(N \sqrt{2 / 3})=V(N U(1))=\frac{4 N^{2}-1}{12} .
$$

Assuming that this is true also for noninteger N's, we get that

$$
V(u)=\frac{6 u^{2}-1}{12}
$$

and therefore that for all $\alpha_{1}, \ldots, \alpha_{k}$,

$$
\xi\left(\alpha_{1}, \ldots, \alpha_{k}\right)=\frac{\left(\sqrt{24 \alpha_{1}+1}+\cdots+\sqrt{24 \alpha_{k}+1}-k\right)^{2}-4}{48} .
$$

This is of course consistent with $\xi\left(1^{\otimes N}\right)=\left(4 N^{2}-1\right) / 12$. It is a nice crosscheck that this fits in nicely with the only rigorously proved value $\xi(2,1)=2$.

Again, this is equivalent to the following formula for the rate function $b_{p}$ as defined in Section 6.2:

$$
b_{p}(a)=\frac{a}{2 a-1}(U(p)-(1 / \sqrt{24}))^{2}+\frac{2 a-1}{48}-\frac{1}{16} .
$$

7.3. Disconnection exponents. Recall that $\eta=V \circ U$. This leads to the following conjecture: for all $\alpha \geq 1$,

$$
\eta(\alpha)=\frac{(\sqrt{24 \alpha+1}-1)^{2}-4}{48} .
$$

This is of course consistent with Duplantier's conjecture $\xi(1,0)=\eta(1)=1 / 4$. It is also consistent with the conjecture $\eta(2)=2 / 3$ which has been shown [19] to be equivalent to Mandelbrot's conjecture [28] that the Hausdorff dimension of the "Brownian frontier" is $4 / 3$. Note again that the exponent $\eta(n)$ is rational only for some integer values of $n$. More precisely, it is very easy to see that for any odd integer $p$ that is not a multiple of 3 , the number 
$n(p):=\left(p^{2}-1\right) / 24$ is an integer and then

$$
\eta(n(p))=\frac{(p-1)^{2}-4}{48}
$$

is a rational number. For all other values of $n, \eta(n)$ is not a rational number. The same remark applies also to the intersection exponents $\xi$ and $\tilde{\xi}$.

Note that these conjectures indicate that some conjectures made by the second author in [30] are false.

Note also that the conjecture for $\eta(5)$ is $\eta(5)=2$. This implies that the problem of existence of exceptional times $t>0$ (if $B$ is a planar Brownian motion) such that:

1. $B_{t}$ is a triple point of $B[0, t]$ (i.e., there exist $0<t_{1}<t_{2}<t$ such that $\left.B_{t_{1}}=B_{t_{2}}=B_{t}\right)$

2. $B_{t}$ belongs to the boundary of the unbounded connected component of the complement of $B[0, t]$ (i.e., $B_{t}$ belongs to the "frontier" of $B[0, t]$ )

is a critical (difficult) problem (see [7]).

8. Conjectures for loop-erased walks. Planar loop-erased random walk is a process derived from simple random walk by erasing loops in chronological order. If $\mathbf{x}=\left[x_{0}, \ldots, x_{n}\right]$ is any finite sequence of points in $\mathbb{Z}^{2}$, we define its loop erasure $L(\mathbf{x})$ in the following way. Let

$$
s_{0}=\sup \left\{j: x_{j}=x_{0}\right\} \text {. }
$$

We define $s_{k}$ inductively by stating that if $s_{k-1}<n$,

$$
s_{k}=\sup \left\{j: x_{j}=x_{s_{k-1}+1}\right\} .
$$

If we let $m$ be the smallest index with $s_{m}=n$, then

$$
L(\mathbf{x})=\left[x_{s_{0}}, \ldots, x_{s_{m}}\right] .
$$

Note that $L(\mathbf{x})$ is a self-avoiding subpath of $\mathbf{x}$ with the same endpoints.

One might hope to define loop-erased Brownian motion in $\mathbb{C}$. For example, consider a grid of points in $\mathbb{C}$ of side $\delta$,

$$
\{\delta j+i \delta k: j, k \in \mathbb{Z}\} .
$$

For each fixed grid we can consider simple random walk taking values in the grid, started, say, on $\{\Re(z)=0\}$ and allowed to run until it reaches $\{\Re(z) \geq n\}$. If we let $\delta \rightarrow 0$, and scale time appropriately, it is well known that the measure on simple random walk paths approaches the measure of Brownian motion. We could also consider conditioned random walks (CRW), conditioned to stay in the set $\{\Re(z)>0\}$ after time 0 , in which case the measure approaches the measure of conditioned Brownian motions (CBM).

For each CRW, we can take the loop erasure of the path. To simplify the statements of this section, we conjecture, but is has not been proved, that there is a limiting measure on paths which we could call conditioned looperased Brownian motion (CLEBM). Proving existence of this measure is an 
open question; in [2,3], it was shown that subsequential limits do exist, but the question of uniqueness is still open. Only in order to simplify the statements of the conjectures in this section, we will assume that this measure exists; in fact, we will even assume that there is an algorithm (possibly a random algorithm) that will take the path of a CBM, $B\left[0, T_{n}\right]$, and produce a subpath $L\left(B\left[0, T_{n}\right]\right)$ which has the measure of CLEBM. For our purposes we will not need to consider the parameterization of the loop-erased path; only the set of points visited by $L\left(B\left[0, T_{n}\right]\right)$ will be relevant.

Assuming the CLEBM exists, there is good reason to believe that it should be conformally invariant (see also Kenyon [13] for a rigorous statement in that direction). Note that if $f$ is a one-to-one function, and $\mathbf{x}=\left[x_{0}, \ldots, x_{n}\right]$ as in (44),

$$
L\left(\left[f\left(x_{0}\right), \ldots, f\left(x_{n}\right)\right]\right)=\left[f\left(x_{s_{0}}\right), \ldots, f\left(x_{s_{m}}\right)\right] .
$$

Hence, even though a conformal mapping can bend and dilate a grid, this change does not affect which points remain in the loop-erased path. We know that Brownian motion is invariant under conformal transformation, and this argument gives reason to believe that the loop-erasing procedure should also be invariant. We will assume that the measure on $L\left(B\left[0, T_{n}\right]\right)$ is conformally invariant. Schramm [32] has recently constructed a random set that should have the same law as $L\left(B\left[0, T_{n}\right]\right)$.

Note that one could state the forthcoming conjectures without making explicit reference to the existence of the scaling limit of loop-erased random walk, and only considering limits of probabilities of nonintersection for loop-erased random walks. We believe that it is more transparent to state the conjectures directly in the continuum.

Let $B^{1}, \ldots, B^{k}$ be independent CBMs starting at $z_{j}=i y_{j}$ where

$$
0<y_{1}<y_{2}<\cdots<y_{k}<\pi \text {. }
$$

If $z \in \mathbb{C}$, we write

$$
\bar{A}=\{z \in \mathbb{C}: z+2 \pi i m \in A \text { for some } m \in \mathbb{Z}\} .
$$

Also we will write

$$
\begin{aligned}
\omega^{j} & =\omega_{n}^{j}=B^{j}\left[0, T_{n}^{j}\right], \\
L\left(\omega^{j}\right) & =L\left(\omega_{n}^{j}\right)=L\left(B^{j}\left[0, T_{n}^{j}\right]\right) .
\end{aligned}
$$

The $k$-point exponent for loop-erased walks, which we will denote as $\zeta_{k}$, is defined by saying that the probability of the event

$$
\overline{L\left(\omega^{j}\right)} \cap \omega^{l}=\varnothing, \quad 1 \leq j<l \leq k
$$

is about $\exp \left(-n \zeta_{k}\right)$. We can think of this recursively. Each time we want to add a path to the configuration, we require that the whole path of the new CBM path not intersect the configuration at that point. If there is no intersection, then we add only the loop erasure of the CBM to the configuration. Note that these exponents correspond (in the discrete setting) to exceptional events for the uniform spanning tree, because this recursive procedure 
is precisely that used by Wilson's algorithm to construct a uniform spanning tree (see [36]).

There is a similar exponent for CBMs restricted to stay in the strip,

$$
\mathscr{T}=\{x+i y: 0<y<\pi\} .
$$

We define $\tilde{\zeta}_{k}$, the $k$-point exponent for the strip, by saying that the probability is about $\exp \left(-n \tilde{\zeta}_{k}\right)$ that (45) occurs and

$$
\omega^{j} \subset \mathscr{T}, \quad j=1, \ldots, k .
$$

Note that we require the entire CBM to lie in $\mathscr{T}$, not just the loop erasure. Also, once we restrict to CBMs that lie in the strip, we can replace $\overline{L\left(\omega^{j}\right)}$ in (45) with $L\left(\omega^{j}\right)$. Duplantier [9], using methods similar to those developed to conjectures (42) and (43), conjectured that

$$
\begin{aligned}
& \zeta_{k}=\frac{k^{2}-1}{4}, \\
& \tilde{\zeta}_{k}=\frac{k^{2}+k}{2} .
\end{aligned}
$$

One value can be made rigorous, at least for the random walk analogue of the exponent (see, e.g., [23]),

$$
\zeta_{3}=2 \text {. }
$$

The exponent $\zeta_{2}$ is related to the growth exponent for loop-erased walks which in turn is related to the dimension of the paths of loop-erased walks. The dimension should be $2-\zeta_{2}$, and the conjecture

$$
\zeta_{2}=\frac{3}{4}
$$

says that the dimension is 5/4. In fact, very recently, Kenyon [14] proved a version of this result for loop-erased walks.

We can also define the exponents $\xi(\lambda)=\xi_{\text {LERW }}(1, \lambda)$ and $\tilde{\xi}(\lambda)=\tilde{\xi}_{\text {LERW }}(1, \lambda)$ for the loop-erased walks by considering the random variables

$$
\begin{aligned}
& Z\left(\omega^{1}\right)=Z_{n}\left(\omega^{1}\right)=\mathbf{P}\left\{\omega^{2} \cap \overline{L\left(\omega^{1}\right)}=\varnothing \mid \omega^{1}\right\}, \\
& \tilde{Z}\left(\omega^{1}\right)=\tilde{Z}_{n}\left(\omega^{1}\right)=\mathbf{P}\left\{\omega^{2} \cap L\left(\omega^{1}\right)=\varnothing, \omega^{2} \subset \mathscr{T} \mid \omega^{1}\right\},
\end{aligned}
$$

and letting

$$
\begin{aligned}
\mathbf{E}\left[Z^{\lambda}\right] & \approx \exp ^{(-n \xi(\lambda))}, \\
\mathbf{E}\left[\tilde{Z}^{\lambda} ; \omega^{1} \subset \mathscr{T}\right] & \approx \exp (-n \tilde{\xi}(\lambda)) .
\end{aligned}
$$

Note that $\xi(1)=\zeta_{2}, \tilde{\xi}(1)=\tilde{\zeta}_{2}$. The behavior at 0 and infinity can be determined easily,

$$
\begin{array}{rlrl}
\xi(0) & =0, & & \tilde{\xi}(0)=1, \\
\xi(\lambda) & \sim \frac{\lambda}{2}, & & \lambda \rightarrow \infty, \\
\tilde{\xi}(\lambda) \sim \lambda, & \lambda \rightarrow \infty .
\end{array}
$$


One value is known rigorously (see [23]), at least for the random walk case,

$$
\xi(3)=2
$$

(there seems to be a trend that if the exponent equals 2 , then there is a way to prove it rigorously). The relation between the $\xi(\lambda), \tilde{\xi}(\lambda)$ and $\zeta_{k}, \tilde{\zeta}_{k}$ comes from the following equations that can be derived in a way similar to the cascade relations for the Brownian motion intersection exponent,

$$
\begin{aligned}
& \zeta_{k+1}=\xi\left(\tilde{\zeta}_{k}\right), \\
& \tilde{\zeta}_{k+1}=\tilde{\xi}\left(\tilde{\zeta}_{k}\right) .
\end{aligned}
$$

Hence, combining (47), (48) and (49) leads to the conjecture

We also let

$$
\xi(\lambda)=\frac{\lambda}{2}+\frac{1}{8}[\sqrt{8 \lambda+1}-1] .
$$

$$
a(\lambda)=a_{\mathrm{LERW}}(\lambda)=\xi^{\prime}(\lambda)=\frac{1}{2}+\frac{1}{2 \sqrt{8 \lambda+1}},
$$

and we define the rate function $b(a)=b_{\mathrm{LERW}}(a)$ by the relation

$$
\mathbf{P}\left\{Z_{n} \approx e^{-n a}\right\} \approx \exp (-n b(a))
$$

(we are not being rigorous in this section, so we will only use this informal definition). If $a$ is in the image of $\xi^{\prime}$ and $\lambda_{a}$ is defined by

$$
\xi^{\prime}\left(\lambda_{a}\right)=a
$$

then

$$
\begin{aligned}
b(a) & =\sup _{\lambda}\{\xi(\lambda)-\lambda a\} \\
& =\xi\left(\lambda_{a}\right)-\lambda_{a} a \\
& =\frac{(a-1)^{2}}{4(2 a-1)} .
\end{aligned}
$$

This last formula may well be equally valid for all $a>1 / 2$.

Similarly, (48) and (50) lead to the conjecture

$$
\tilde{\xi}(\lambda)=\lambda+1+\frac{1}{2}[\sqrt{8 \lambda+1}-1] .
$$

Note that this is consistent with (48) and (50). The $\lambda+1$ term comes from the probability that (46) holds. We define the exponent,

$$
\hat{\xi}(\lambda)=\hat{\xi}_{\text {LERW }}(1, \lambda)=\tilde{\xi}(\lambda)-\lambda-1,
$$

to be the corresponding exponent for paths that are conditioned to satisfy (46). Then the conjecture becomes

We then get

$$
\hat{\xi}(\lambda)=\frac{1}{2}[\sqrt{8 \lambda+1}-1] .
$$

$$
\hat{a}(\lambda)=\hat{a}_{\mathrm{LERW}}(\lambda)=\hat{\xi}^{\prime}(\lambda)=\frac{2}{\sqrt{8 \lambda+1}},
$$


and if we define $\hat{b}$ similarly, we get the conjecture

$$
\hat{b}(a)=\frac{(2-a)^{2}}{a} \text {. }
$$

Let us now write

and

$$
\xi(1, \lambda)=\xi(\lambda), \quad \tilde{\xi}(1, \lambda)=\tilde{\xi}(\lambda)
$$

$$
\xi\left(1^{\otimes k}\right)=\zeta_{k}, \quad \tilde{\xi}\left(1^{\otimes k}\right)=\tilde{\zeta}_{k} .
$$

It is not difficult to define

$$
\xi\left(1^{\otimes k}, \lambda\right), \quad \tilde{\xi}\left(1^{\otimes k}, \lambda\right),
$$

but at the moment we have no interpretation for

$$
\xi\left(p_{1}, \ldots, p_{k}\right), \quad \tilde{\xi}\left(p_{1}, \ldots, p_{k}\right),
$$

even for positive integer $p_{1}, \ldots, p_{k}$. A conformal invariance argument suggests the cascade relation

$$
\xi\left(1^{\otimes k+l}, \lambda\right)=\xi\left(1^{\otimes k}, \tilde{\xi}\left(1^{\otimes l}, \lambda\right)\right)
$$

holds. This cascade relation gives us an interesting consistency check for our conjectures which we only sketch here. The triple point equality,

$$
\xi(1,1,1)=2,
$$

is derived by considering the first hitting of a loop-erased walk by a simple random walk. As part of the proof, one sees that for a typical configuration satisfying (45),

$$
L\left(\omega^{1}\right) \cup L\left(\omega^{2}\right)
$$

looks like the "middle" of a single loop-erased walk and $\omega^{3}$ is required to miss the walk. By the relation between escape probabilities and harmonic measure, the conditional distribution of

$$
L\left(\omega^{1}\right) \cup L\left(\omega^{2}\right)
$$

under this conditioning should be approximately the harmonic measure of this single loop-erased walk. By Makarov's theorem [16], [27], the harmonic measure should be carried on a set of dimension 1 ; here that translates to the fact that in a typical configuration of $\left(\omega^{1}, \omega^{2}, \omega^{3}\right)$, the probability that a fourth walker $\omega^{4}$ will miss $L\left(\omega^{1}\right) \cup L\left(\omega^{2}\right)$ should look like $e^{-n}$. This translates to

$$
\left.\frac{\partial \xi(1,1, \lambda)}{\partial \lambda}\right|_{\lambda=1}=1 .
$$

Using the relation $\xi(1,1, \lambda)=\xi(1, \tilde{\xi}(1, \lambda))$, we can check that the conjectured values for $\xi$ and $\tilde{\xi}$ satisfy this. This is analogous to the rigorous argument for the Brownian motion exponent to show that the derivative with respect to $\lambda$ of $\xi_{2}(2, \lambda)$, evaluated at $\lambda=1$, is 1 . 


\section{REFERENCES}

[1] Ahlfors, L. V. (1973). Conformal Invariants: Topics in Geometric Function Theory. McGraw-Hill, New York.

[2] Aizenman, M. and Burchard, A. (1999). Hölder regularity and dimension bounds for random curves. Duke Math. J. 99 419-453.

[3] Aizenman, M., Burchard, A., Newman, C. and Wilson, D. (1999). Scaling limits for minimal and random spanning trees in two dimensions. Random Structures Algorithms. To appear.

[4] Burdzy, K. and LAwler, G. F. (1990). Non-intersection exponents for random walk and Brownian motion I: Existence and an invariance principle. Probab. Theory Related Fields 84 393-410.

[5] Burdzy, K. and LAwler, G. F. (1990). Non-intersection exponents for random walk and Brownian motion II: Estimates and applications to a random fractal. Ann. Probab. 18 981-1009.

[6] Burdzy, K., Lawler, G. F. and Polaski, T. (1989). On the critical exponent for random walk intersections. J. Statist. Phys. 56 1-12.

[7] Burdzy, K. and Werner, W. (1996). No triple point of planar Brownian motion is accessible. Ann. Probab. 24 125-147.

[8] Cranston, M. and Mountford, T. (1991). An extension of a result by Burdzy and Lawler. Probab. Theory Related Fields 89 487-502.

[9] Duplantier, B. (1992). Loop-erased self-avoiding walks in two dimensions: exact critical exponents and winding numbers. Phys. A 191 516-522.

[10] Duplantier, B. and Kwon, K.-H. (1988). Conformal invariance and intersection of random walks. Phys. Rev. Lett. $612514-2517$.

[11] Duplantier, B., Lawler, G. F., Le Gall, J.-F. and Lyons, T. J. (1993). The geometry of the Brownian curve. Bull. Sci. Math. 2 117 91-106.

[12] Goddard, P. and D. Olive, eds. (1988). Kac-Moody and Virasoro Algebras. World Scientific, Singapore.

[13] KENYON, R. (1998). Conformal invariance of domino tiling. Preprint.

[14] Kenyon, R. (1998). The asymptotic determinant of the discrete Laplacian. Preprint.

[15] LAWLER, G. F. (1991). Intersections of Random Walks. Birkhäuser, Boston.

[16] Lawler, G. F. (1993). A discrete analogue of a theorem of Makarov. Combin. Probab. Comput. 2 181-200.

[17] LAwler, G. F. (1995). Hausdorff dimension of cut points for Brownian motion. Electron. J. Probab. 1 1-20.

[18] LAwler, G. F. (1996). The dimension of the frontier of planar Brownian motion. Electron. Comm. Probab. 1 29-47.

[19] Lawler, G. F. (1997). Cut times for simple random walk. Electron. J. Probab. 1 1-24.

[20] LAwler, G. F. (1997). The frontier of a Brownian path is multifractal. Preprint.

[21] LAwler, G. F. (1998). Strict concavity of the intersection exponent for Brownian motion in two and three dimensions. Math. Phys. Electron. J. 4 1-67.

[22] Lawler, G. F. (1999). Loop-erased random walk. In Perplexing Problems in Probability: Volume in honor of Harry Kesten. Birkhäuser, Boston. To appear.

[23] Lawler, G. F. and Puckette, E. E. (1997). The disconnection exponent for simple random walk. Israel J. Math. 99 109-122.

[24] Lawler, G. F. and Puckette, E. E. (1998). The intersection exponent for simple random walk. Preprint.

[25] LI, B. and SokAL, A. D. (1990). High-precision Monte Carlo test of the conformal invariance predictions for two-dimensional mutually avoiding walks. J. Statist. Phys. 61 723-748.

[26] Makarov, N. G. (1985). Distortion of boundary sets under conformal mapping. Proc. London Math. Soc. 51 369-384.

[27] Mandelbrot, B. B. (1982). The Fractal Geometry of Nature. Freeman, San Francisco. 
[28] Port, S. C. and Stone, C. J. (1978). Brownian Motion and Classical Potential Theory. Academic Press, New York.

[29] Puckette, E. E. and Werner, W. (1996). Monte-Carlo tests and conjectures for disconnection exponents. Electron. Comm. Probab. 1 49-64.

[30] Revuz, D. and Yor, M. (1991). Continuous Martingales and Brownian Motion. Springer, Berlin.

[31] Schramm, O. (1999). Scaling limits of loop-erased random walks and uniform spanning trees Israel J. Math. To appear.

[32] Werner, W. (1995). On Brownian disconnection exponents. Bernoulli 1 371-380.

[33] Werner, W. (1996). Bounds for disconnection exponents. Electron. Comm. Probab. 1 19-28.

[34] Werner, W. (1997). Asymptotic behaviour of disconnection and nonintersection exponents. Probab. Theory Related Fields 108 131-152.

[35] Wilson, D. B. (1996). Generating random trees more quickly than the cover time. ACM Symp. Theory of Computing 296-303.

DEPARTMENT OF MATHEMATics

Box 90320

DUKE UNIVERSITY

DURHAM, NORTH CAROLINA 27708-0320

E-MAIL: jose@math.duke.edu
DÉPARTEMENT De Mathématiques

BÂT. 425

UnIVERSITÉ PARIS-SUd

91405 ORSAY CEDEX

FRANCE

E-MAIL: wendelin.werner@math.u-psud.fr 